\title{
THE ALPERIN WEIGHT CONJECTURE AND DADE'S CONJECTURE FOR THE SIMPLE GROUP J 4
}

\author{
JIANBEI AN, E. A. O’BRIEN AND R. A. WILSON
}

\begin{abstract}
The authors construct faithful permutation representations of maximal 2-local subgroups and classify the radical chains of the Janko simple group $\mathrm{J}_{4}$; hence the Alperin weight conjecture and the Dade reductive conjecture for $\mathrm{J}_{4}$ are verified.
\end{abstract}

\section{Introduction}

The program of deciding Dade's reductive conjecture [10] for the sporadic groups has made very substantial progress: it has been verified for all of the sporadic simple groups except $\mathrm{Fi}_{24}^{\prime}, \mathrm{J}_{4}, \mathrm{~B}$ and $\mathrm{M}$.

The use of computer algebra systems, namely MAGMA [7] and GAP [12], to study permutation (or in some cases matrix) representations of the groups has been a central step of the program. Since the smallest faithful permutation representation of $\mathrm{J}_{4}$ has degree 173067389 , it is difficult to verify the conjecture directly. However, from the classification of maximal subgroups of $\mathrm{J}_{4}$ (see [14]), we know that the normalizer of each radical 2- and 3 -subgroup of $\mathrm{J}_{4}$ is a subgroup of one of precisely four maximal 2-local subgroups. Thus we can classify radical chains in these four maximal subgroups without performing any calculation in $\mathrm{J}_{4}$.

In this paper, we construct faithful permutation representations for each maximal 2-local subgroup, classify radical chains, and hence verify the Alperin weight conjecture and the Dade reductive conjecture for $\mathrm{J}_{4}$.

Let $G$ be a finite group, $p$ a prime and $B$ a $p$-block of $G$. Alperin [1] conjectured that the number of $B$-weights equals the number of irreducible Brauer characters of $B$. Subsequently, Dade [9] generalized the Knörr-Robinson version of the Alperin weight conjecture, and presented his ordinary conjecture, exhibiting the number of ordinary irreducible characters of a fixed defect in $B$ in terms of an alternating sum of related values for $p$-blocks of some $p$-local subgroups of $G$. Later, Dade [10] announced that his reductive conjecture needs only to be verified for finite non-abelian simple groups; in addition, if a finite group has a trivial Schur multiplier and trivial outer automorphism group, then the ordinary conjecture is equivalent to the reductive conjecture.

The paper is organized as follows. In Section 2, we fix the notation, state the conjectures in detail, and state two lemmas. In Section 3, we explain how to construct faithful permutation representations of the four maximal 2-local subgroups. In Section 4, we recall the modified local strategy $[3,4]$; we also explain how we applied it to determine the radical subgroups of each maximal subgroup, and how to fuse the radical subgroups in $\mathrm{J}_{4}$. In Section 5, we use the list of radical subgroups of $\mathrm{J}_{4}$ given by [17] to verify the Alperin weight conjecture.

This work was supported by the Marsden Fund of New Zealand via grant \#9144/3368248, and by an International Research Collaboration Visit grant from the University of Auckland.

Received 6 February 2003, revised 5 March 2003; published 1 August 2003.

2000 Mathematics Subject Classification 20C20, 20C34, 20 D08

(C) 2003, Jianbei An, E. A. O’Brien and R. A. Wilson 
In Section 6, we do some cancellations in the alternating sum of Dade's conjecture, and then determine radical chains (up to conjugacy) and their local structures. Finally, we verify the ordinary conjecture of Dade for $\mathrm{J}_{4}$.

\section{Dade's ordinary conjecture}

Let $R$ be a $p$-subgroup of a finite group $G$. Then $R$ is radical if $O_{p}(N(R))=R$, where $O_{p}(N(R))$ is the largest normal $p$-subgroup of the normalizer $N(R)=N_{G}(R)$. Denote by $\operatorname{Irr}(G)$ the set of all irreducible ordinary characters of $G$, and let $\operatorname{Blk}(G)$ be the set of $p$ blocks, $B \in \operatorname{Blk}(G)$ and $\varphi \in \operatorname{Irr}(N(R) / R)$. The pair $(R, \varphi)$ is called a $B$-weight if $\mathrm{d}(\varphi)=0$ and $B(\varphi)^{G}=B$ (in the sense of Brauer), where $\mathrm{d}(\varphi)=\log _{p}\left(|G|_{p}\right)-\log _{p}\left(\varphi(1)_{p}\right)$ is the $p$-defect of $\varphi$ and $B(\varphi)$ is the block of $N(R)$ containing $\varphi$. A weight is always identified with its $G$-conjugates. Let $\mathcal{W}(B)$ be the number of $B$-weights, and let $\ell(B)$ be the number of irreducible Brauer characters of $B$. Alperin conjectured that $\mathcal{W}(B)=\ell(B)$ for each $B \in \operatorname{Blk}(G)$.

Given a $p$-subgroup chain

$$
C: P_{0}<P_{1}<\ldots<P_{n}
$$

of $G$, define $|C|=n, C_{k}: P_{0}<P_{1}<\ldots<P_{k}$, and

$$
N(C)=N_{G}(C)=N\left(P_{0}\right) \cap N\left(P_{1}\right) \cap \ldots \cap N\left(P_{n}\right) .
$$

The chain $C$ is radical if it satisfies the following two conditions:

(a) $P_{0}=O_{p}(G)$; and

(b) $P_{k}=O_{p}\left(N\left(C_{k}\right)\right)$ for $1 \leqslant k \leqslant n$.

Denote by $\mathcal{R}=\mathcal{R}(G)$ the set of all radical $p$-chains of $G$.

Let $\mathrm{k}\left(N_{G}(C), B, d\right)$ be the number of characters $\psi$ in $\operatorname{Irr}\left(N_{G}(C)\right)$ such that $\mathrm{d}(\psi)=d$ and $B(\psi)^{G}=B$. In the notation used above, the Dade ordinary conjecture is stated as follows.

DADE's ORDiNARY CONJECTURE (see [9]). If $O_{p}(G)=1$ and $B$ is a p-block of $G$ with defect group $D(B) \neq 1$, then for any integer $d \geqslant 0$,

$$
\sum_{C \in \mathcal{R} / G}(-1)^{|C|} \mathrm{k}\left(N_{G}(C), B, d\right)=0,
$$

where $\mathcal{R} / G$ is a set of representatives for the $G$-orbits of $\mathcal{R}$.

Let $G$ be the Janko simple group $\mathrm{J}_{4}$. Then its Schur multiplier and outer automorphism group are both trivial, so by [10], Dade's ordinary conjecture is equivalent to his reductive conjecture.

In Section 6, we shall use the following lemmas.

Lemma 2.1. Let $\sigma: O_{p}(G)<P_{1}<\ldots<P_{m-1}<Q=P_{m}<P_{m+1}<\ldots<P_{\ell}$ be $a$ fixed radical $p$-chain of a finite group $G$, where $1 \leqslant m<\ell$. Suppose that

$$
\sigma^{\prime}: O_{p}(G)<P_{1}<\ldots<P_{m-1}<P_{m+1}<\ldots<P_{\ell}
$$

is also a radical p-chain such that $N_{G}(\sigma)=N_{G}\left(\sigma^{\prime}\right)$. Let $\mathcal{R}^{-}(\sigma, Q)$ be the subfamily of $\mathcal{R}(G)$ consisting of chains $C$ whose $(\ell-1)$ th subchain $C_{\ell-1}$ is conjugate to $\sigma^{\prime}$ in $G$, and 
$\mathcal{R}^{0}(\sigma, Q)$ the subfamily of $\mathcal{R}(G)$ consisting of chains $C$ whose $\ell$ th subchain $C_{\ell}$ is conjugate to $\sigma$ in $G$. Then the map $g$, sending any

$$
O_{p}(G)<P_{1}<\ldots<P_{m-1}<P_{m+1}<\ldots<P_{\ell}<\ldots
$$

in $\mathcal{R}^{-}(\sigma, Q)$ to

$$
O_{p}(G)<P_{1}<\ldots<P_{m-1}<Q<P_{m+1}<\ldots<P_{\ell}<\ldots,
$$

induces a bijection, denoted again by $g$, from $\mathcal{R}^{-}(\sigma, Q)$ onto $\mathcal{R}^{0}(\sigma, Q)$. Moreover, for any $C$ in $\mathcal{R}^{-}(\sigma, Q)$, we have $|C|=|g(C)|-1$ and $N_{G}(C)=N_{G}(g(C))$.

Proof. This is straightforward.

LEMMA 2.2. If $Q$ is a $p$-subgroup of a finite group $G$, then there is a radical $p$-subgroup $R$ such that

$$
Q \leqslant R \quad \text { and } \quad N_{G}(Q) \leqslant N_{G}(R) .
$$

Proof. This follows by [2, Lemma 2.1].

\section{Construction of permutation representations of maximal 2-local subgroups}

We use the notation of [8]. In particular, $p^{1+2 \gamma}=p_{+}^{1+2 \gamma}$ is an extra-special group of order $p^{1+2 \gamma}$ with exponent $p$ or type + according to whether $p$ is odd or even. If $X$ and $Y$ are groups, we use $X \cdot Y$ and $X: Y$ to denote a nonsplit extension and a split extension of $X$ by $Y$, respectively. Given a positive integer $n$, we use $p^{n}$ to denote the elementary abelian group of order $p^{n}, n$ to denote the cyclic group of order $n$, and $D_{2 n}$ to denote the dihedral group of order $2 n$.

The four maximal 2-local subgroups of $\mathrm{J}_{4}$ that we wish to construct are $2^{11}: M_{24}$, $2^{10}: L_{5}(2), 2^{3+12} \cdot\left(S_{5} \times L_{3}(2)\right)$ and $2^{1+12} \cdot 3 \cdot M_{22}: 2$. The first two of these are easy to construct abstractly as affine groups. The first can be written as $12 \times 12$ matrices over $G F(2)$, acting either with a fixed vector or with a fixed hyperplane. These representations can be easily obtained from a submodule or quotient module (respectively) of the restriction of the 112-dimensional representation of $\mathrm{J}_{4}$. These give rise naturally to permutation representations on $759+1288+2048$ non-zero vectors in the one case, or $1+1518+2576$ in the second. Any of the three faithful representations, of degrees 2048, 1518, and 2576, can then be used to generate the character table of the group.

Similarly, the group $2^{10}: L_{5}(2)$ has two natural affine representations, in which the orbits of non-zero vectors are either $155+868+1024$ or $1+310+1736$ in length, and again any of the three faithful representations, of degree 1024, 310 and 1736, can be used as a starting point for the calculations.

The other two maximal 2-local subgroups of $\mathrm{J}_{4}$ are harder to construct. They can be obtained as subgroups of $\mathrm{J}_{4}$ using the words in the standard generators of $\mathrm{J}_{4}$ given in [5]. This, however, limits us to two particular representations of the groups, given by restricting the representations of $\mathrm{J}_{4}$ in dimension 112 over $G F(2)$ or dimension 1333 over $G F(11)$. For the purposes of calculating the character tables, we want to obtain faithful permutation representations on a reasonably small number of points.

On the other hand, of course, the point stabilizer in a faithful representation cannot contain any non-trivial subgroup that is normal in the whole group, and this puts severe constraints on which subgroups we can use as a point stabilizer. For example, in the case $2^{1+12} \cdot 3 \cdot M_{22}: 2$, if we are to avoid the central involution, the point stabilizer can have at most 
a subgroup of order $2^{6}$ in the normal $2^{1+12}$. Moreover, such a subgroup must be invariant under a reasonable subgroup of $6 \cdot M_{22}$, which must again split off the central involution. The biggest subgroup that we could identify as a potential point stabilizer was a group of shape $2^{6}: 3 \cdot A_{6}$, which we found as the subgroup generated by certain words in the given generators of $2^{1+12} \cdot 3 \cdot M_{22}: 2$.

This subgroup has index $2^{7} \cdot 2.77 .16=630784$. We obtained the corresponding permutation representation on 630784 points by calculating the subgroup explicitly in the 112-dimensional representation over $G F(2)$, and using MAGMA to calculate the permutation action on the cosets. We then found that in fact there is a block system of 315392 blocks of size 2, such that the action on the blocks is still faithful. This representation was then used as input to the next stage of calculation.

In the remaining case, we found a subgroup of shape $2^{1+8}\left(5 \times S_{4}\right)$ as a potential point stabilizer in $2^{3+12}\left(S_{5} \times L_{3}(2)\right)$. We used the 1333-dimensional $G F(11)$-representation of $\mathrm{J}_{4}$, and restricted to a 1120-dimensional submodule on which the maximal 2-local subgroup acts faithfully and irreducibly. Restricting further to the potential point stabilizer, we found a 15-dimensional invariant subspace. Taking the permutation action of our maximal 2-local subgroup on the 61440 images of this 15-space gave us the desired faithful permutation representation.

\section{A local subgroup strategy and fusions}

Kleidman and Wilson [14] classified the maximal subgroups of $\mathrm{J}_{4}$. From this, we know that there are 4 maximal 2-local subgroups up to conjugacy. In addition, each radical 2- and 3 -subgroup $R$ of $\mathrm{J}_{4}$ is radical in one of the subgroups $M$ and, further, $N_{\mathrm{J}_{4}}(R)=N_{M}(R)$. The radical 2-subgroups of $\mathrm{J}_{4}$ are classified by [17].

In [3] and [4], a (modified) local strategy was developed to classify the radical $p$ subgroups $R$. We review this method here.

Let $Q=O_{p}(M)$, so that $Q \leqslant R$. Choose a subgroup $X$ of $M$. We explicitly compute the coset action of $M$ on the cosets of $X$ in $M$; we obtain a group $W$ representing this action, a group homomorphism $f$ from $M$ to $W$, and the kernel $K$ of $f$. For a suitable $X$, we have $K=Q$ and the degree of the action of $W$ on the cosets is much smaller than that of $M$. We can now directly classify the radical $p$-subgroup classes of $W$, and the preimages in $M$ of the radical subgroup classes of $W$ are the radical subgroup classes of $M$.

After applying the strategy, we list the radical subgroups of each $M$, and then we do the fusions as follows.

Suppose that $R$ is a radical $p$-subgroup of $M$. Using the local structure, we can determine whether or not $N_{M}(R)$ is a subgroup of another maximal subgroup $M^{\prime}$. Suppose that $N_{M}(R)$ is a subgroup of $M^{\prime}$. By Lemma 2.2, there is a radical subgroup $R^{\prime}$ of $M^{\prime}$ such that $R \leqslant R^{\prime}$ and $N_{M}(R) \leqslant N_{M^{\prime}}\left(R^{\prime}\right)$. Using the local structure, we can determine whether or not $R$ is radical in $M^{\prime}$; if so, we can identify $R$ with a radical subgroup $R^{\prime}$ of $M^{\prime}$. In this case, $N_{M}(R)={ }_{G} N_{M^{\prime}}\left(R^{\prime}\right)$. Some more details are given below.

The computations reported in this paper were carried out using MAGMA V2.9-9 on a Sun UltraSPARC Enterprise 4000 server.

\section{Weights}

Let $\mathcal{R}_{0}(G, p)$ be a set of representatives for conjugacy classes of radical $p$-subgroups of $G$. For $H, K \leqslant G$, we write $H \leqslant G K$ if $x^{-1} H x \leqslant K$, and we write $H \in_{G} \mathcal{R}_{0}(G, p)$ if $x^{-1} H x \in \mathcal{R}_{0}(G, p)$ for some $x \in G$. 
The Alperin weight conjecture and Dade's conjecture for the simple group $\mathrm{J}_{4}$

Let $G$ be the Janko simple group $\mathrm{J}_{4}$. Then

$$
|G|=2^{21} \cdot 3^{3} \cdot 5 \cdot 7 \cdot 11^{3} \cdot 23 \cdot 29 \cdot 31 \cdot 37 \cdot 43,
$$

and we may suppose that $p \in\{2,3,11\}$, since both conjectures hold for a block with a cyclic defect group, by [9, Theorem 7.1]. If $p=11$, then by [6, Proposition 1.3], a Sylow subgroup of $G$ is a trivial intersection group, so that Dade's ordinary conjecture and Alperin's weight conjecture follow, by [11]. Moreover, Uno's ordinary conjecture, which is refinement of Dade's ordinary conjecture, also holds for $J_{4}$. Thus we may suppose that $p=2$ or $p=3$.

We denote by $\operatorname{Irr}^{0}(H)$ the set of ordinary irreducible characters of $p$-defect 0 of a finite group $H$, and by $\mathrm{d}(H)$ the number $\log _{p}\left(|H|_{p}\right)$. Given $R \in \mathcal{R}_{0}(G, p)$, let $C(R)=C_{G}(R)$ and $N=N_{G}(R)$. If $B_{0}=B_{0}(G)$ is the principal $p$-block of $G$, then (see [3, (4.1)])

$$
\mathcal{W}\left(B_{0}\right)=\sum_{R}\left|\operatorname{Irr}^{0}(N / C(R) R)\right|,
$$

where $R$ runs over the set $\mathcal{R}_{0}(G, p)$ such that $\mathrm{d}(C(R) R / R)=0$. The character table of $N / C(R) R$ can be calculated by MAGMA, and so we find that $\left|\operatorname{Irr}^{0}(N / C(R) R)\right|$.

In Table 1, we recall the classification from [17] of the radical 2-subgroups of $G=\mathrm{J}_{4}$.

Suppose that $p=3$. As shown in [14, Section 3],

$$
\mathcal{R}_{0}(G, 3)=\left\{1,3,3^{2}, 3_{+}^{1+2}\right\},
$$

where $3=Z\left(3_{+}^{1+2}\right)$. In addition, $C(3)=6 \cdot M_{22}, C\left(3^{2}\right)=3^{2} \times 2^{3}, C\left(3_{+}^{1+2}\right)=6$ and

$$
N(R)= \begin{cases}6 \cdot M_{22}: 2 \leqslant 2_{+}^{1+12} \cdot 3 \cdot M_{22}: 2, & \text { if } R=3, \\ \left(3^{2}: 2 \times 2^{3}\right) \cdot S_{4} \leqslant 2^{11}: M_{24}, & \text { if } R=3^{2}, \\ \left(2 \times 3_{+}^{1+2}: 8\right): 2 \leqslant 2_{+}^{1+12} \cdot 3 \cdot M_{22}: 2, & \text { if } R=3_{+}^{1+2} .\end{cases}
$$

Lemma 5.1. Let $G=\mathrm{J}_{4}$ and $B_{0}=B_{0}(G)$, let $\mathrm{Blk}^{+}(G, p)$ be the set of p-blocks with a nontrivial defect group, and let $\operatorname{Irr}^{+}(G)$ be the characters of $\operatorname{Irr}(G)$ with positive p-defect. If a defect group $D(B)$ of $B$ is cyclic, then $\operatorname{Irr}(B)$ is given by [13, p. 326].

(a) If $p=3$, then $\mathrm{Blk}^{+}(G, p)=\left\{B_{i} \mid 0 \leqslant i \leqslant 6\right\}$ such that $D\left(B_{1}\right) \simeq 3_{+}^{1+2}, D\left(B_{2}\right) \simeq 3^{2}$ and $D\left(B_{i}\right) \simeq 3$ for $3 \leqslant i \leqslant 6$. In the notation of $[8$, p. 188],

$$
\begin{aligned}
& \operatorname{Irr}(B)=\left\{\chi_{2}, \chi_{3}, \chi_{12}, \chi_{13}, \chi_{17}, \chi_{18}, \chi_{22}, \chi_{23}, \chi_{24}, \chi_{26}, \chi_{38}, \chi_{39}, \chi_{44}, \chi_{50}\right\}, \\
& \operatorname{Irr}\left(B_{2}\right)=\left\{\chi_{14}, \chi_{21}, \chi_{25}, \chi_{27}, \chi_{28}, \chi_{30}, \chi_{31}, \chi_{35}, \chi_{41}\right\},
\end{aligned}
$$

and

$$
\operatorname{Irr}\left(B_{0}\right)=\operatorname{Irr}^{+}(G) \backslash\left(\cup_{i=1}^{6} \operatorname{Irr}\left(B_{i}\right)\right) .
$$

Moreover, $\ell\left(B_{0}\right)=\ell\left(B_{1}\right)=9, \ell\left(B_{2}\right)=5, \ell\left(B_{i}\right)=2$ for $3 \leqslant i \leqslant 5$ and $\ell\left(B_{6}\right)=1$.

(b) If $p=2$, then $\mathrm{Blk}^{+}(G, 2)=\left\{B_{0}\right\}$, and so $\operatorname{Irr}\left(B_{0}\right)=\operatorname{Irr}^{+}(G)$. Moreover, $\ell\left(B_{0}\right)=22$.

Proof. If $B \in \operatorname{Blk}(G, p)$ is non-principal with $D=D(B)$, then $\operatorname{Irr}^{0}(C(D) D / D)$ has a non-trivial character $\theta$ and $N(\theta) / C(D) D$ is a $p^{\prime}$-group, where $N(\theta)$ is the stabilizer of $\theta$ in $N(D)$. By $\left[13\right.$, p. 326], we may suppose that $D$ is non-cyclic. Thus $D=3^{2}$ or $3_{+}^{1+2}$. In each case, $N(D)$ has one orbit on the non-trivial character $\operatorname{ofr}^{0}(C(D) D / D)$ with $N(\theta) / C(D) D$ a $3^{\prime}$-group. 
The Alperin weight conjecture and Dade's conjecture for the simple group $\mathrm{J}_{4}$

Table 1: Non-trivial radical 2-subgroups of $\mathbf{J}_{4}$

\begin{tabular}{|c|c|c|c|}
\hline$R$ & $C(R)$ & $N(R)$ & $\left|\operatorname{Irr}^{0}(N / C(R) R)\right|$ \\
\hline $2^{10}$ & $2^{10}$ & $2^{10}: L_{5}(2)$ & 1 \\
\hline $2^{11}$ & $2^{11}$ & $2^{11}: M_{24}$ & 0 \\
\hline $2^{1+12}$ & 2 & $2^{1+12} \cdot\left(3 \cdot M_{22}\right): 2$ & 1 \\
\hline $2^{10}: 2^{4}$ & $2^{4}$ & $2^{10}: 2^{4} \cdot L_{4}(2)$ & 1 \\
\hline $2^{11}: 2^{4}$ & $2^{6}$ & $2^{11}: 2^{4} \cdot A_{8}$ & 1 \\
\hline $2^{3+12}$ & $2^{3}$ & $2^{3+12} \cdot\left(S_{5} \times L_{3}(2)\right)$ & 0 \\
\hline $2^{3+12} \cdot 2$ & $2^{3}$ & $2^{3+12} \cdot 2\left(S_{3} \times L_{3}(2)\right)$ & 1 \\
\hline $2^{1+12} \cdot 2^{3}$ & 2 & $2^{1+12} \cdot 2^{3} \cdot\left(S_{3} \times L_{3}(2)\right)$ & 1 \\
\hline $2^{3} \cdot 2^{6+8}$ & $2^{3}$ & $2^{3} \cdot 2^{6+8} \cdot\left(S_{3} \times L_{3}(2)\right)$ & 1 \\
\hline $2^{2} \cdot 2^{5+10}$ & $2^{2}$ & $2^{2} \cdot 2^{5+10} \cdot\left(S_{3} \times S_{5}\right)$ & 0 \\
\hline $2^{1+12} \cdot 2^{4}$ & 2 & $2^{1+12} \cdot 2^{4} \cdot 3 \cdot S_{6}$ & 1 \\
\hline $2^{3+12} \cdot 2^{2}$ & 2 & $2^{2+12} \cdot 2^{2} \cdot\left(S_{3} \times S_{5}\right)$ & 0 \\
\hline $2^{10} \cdot 2^{3+4}$ & 2 & $2^{10} \cdot 2^{3+4} \cdot L_{3}(2)$ & 1 \\
\hline $2^{3+12} \cdot D_{8}$ & $2^{3}$ & $2^{3+12} \cdot D_{8} \cdot L_{3}(2)$ & 1 \\
\hline $2^{3+12} \cdot 2^{3}$ & $2^{2}$ & $2^{3+12} \cdot 2^{3} \cdot\left(S_{3} \times S_{3}\right)$ & 1 \\
\hline $2^{1+12} \cdot 2^{2+3}$ & 2 & $2^{1+12} \cdot 2^{2+3} \cdot\left(S_{3} \times S_{3}\right)$ & 1 \\
\hline $2^{11} \cdot 2^{1+6}$ & $2^{3}$ & $2^{11} \cdot 2^{1+6} \cdot L_{3}(2)$ & 1 \\
\hline $2^{1+12} \cdot 2^{5}$ & 2 & $2^{1+12} \cdot 2^{5} \cdot S_{5}$ & 0 \\
\hline $2^{1+12} \cdot 2^{2} \cdot 2^{4}$ & 2 & $2^{1+12} \cdot 2^{2} \cdot 2^{4} \cdot\left(S_{3} \times S_{3}\right)$ & 1 \\
\hline $2^{1+12} \cdot 2 \cdot 2^{2+3}$ & 2 & $2^{1+12} \cdot 2 \cdot 2^{2+3} \cdot\left(S_{3} \times S_{3}\right)$ & 1 \\
\hline $2^{3+12} \cdot 2^{4}$ & 2 & $2^{3+12} \cdot 2^{4} \cdot\left(S_{3} \times S_{3}\right)$ & 1 \\
\hline $2^{1+12} \cdot 2^{3} \cdot 2^{3}$ & 2 & $2^{1+12} \cdot 2^{3} \cdot 2^{3} \cdot S_{3}$ & 1 \\
\hline $2^{3+12} \cdot 2^{3} \cdot 2^{2}$ & $2^{2}$ & $2^{3+12} \cdot 2^{3} \cdot 2^{2} \cdot S_{3}$ & 1 \\
\hline $2^{11} \cdot 2^{2} \cdot 2^{3} \cdot 2^{4}$ & 2 & $2^{11} \cdot 2^{2} \cdot 2^{3} \cdot 2^{4} \cdot S_{3}$ & 1 \\
\hline $2^{1+12} \cdot 2^{2+5}$ & 2 & $2^{1+12} \cdot 2^{2+5} \cdot S_{3}$ & 1 \\
\hline $2^{1+12} \cdot 2 \cdot 2^{3} \cdot 2^{3}$ & 2 & $2^{1+12} \cdot 2 \cdot 2^{3} \cdot 2^{3} \cdot S_{3}$ & 1 \\
\hline$S$ & 2 & $S$ & 1 \\
\hline
\end{tabular}


Using the method of central characters, $\operatorname{Irr}(B)$ is as above. If $D(B)$ is cyclic, then $\ell(B)$ is given by [13, p. 326].

If $p=3$ and $B=B_{1}$ or $B=B_{2}$, then the non-trivial elements of $D(B)$ are of type $3 A$, and $C_{G}(3 A)=6 . M_{22}$. It follows by [15, Theorem 5.4.13] that

$$
k(B)=\ell(B)+\sum_{b \in \operatorname{Blk}\left(6 . M_{22}, B\right)} \ell(b),
$$

where $\operatorname{Blk}\left(6 \cdot M_{22}, B\right)=\left\{b \in \operatorname{Blk}\left(6 \cdot M_{22}\right): b^{G}=B\right\}$.

If $B=B_{2}$, then $D\left(B_{2}\right)={ }_{G} 3^{2}, \operatorname{Blk}\left(6 . M_{22}, B\right)=\left\{b_{2}, b_{2}^{\prime}\right\}$ and, by [13, p. 78], $\ell\left(b_{2}\right)=$ $\ell\left(b_{2}^{\prime}\right)=2$, and so $\ell\left(B_{2}\right)=5$.

If $B=B_{1}$, then $D\left(B_{1}\right)={ }_{G} 3_{+}^{1+2}, \operatorname{Blk}\left(6 . M_{22}, B\right)=\{b\}$; moreover, $\ell(b)=\ell\left(b_{1}\right)$ for a unique block of $2 . M_{22}$ with $D\left(b_{1}\right) \simeq 3^{2}$. Now $2 . M_{22}$ has exactly one class $x$ of elements of order 3 and $C_{2 . M_{22}}(x)=3^{2} \times 2^{3}$. It follows, by [15, Theorem 5.4.13] again, that $\ell\left(b_{1}\right)=k\left(b_{1}\right)-1=6-1=5$, so that $\ell\left(B_{1}\right)=14-5=9$.

If $\ell_{p}(G)$ is the number of $p$-regular $G$-conjugacy classes, then $\ell_{3}(G)=43$ and $\ell_{2}(G)=$ 25 . Thus $\ell\left(B_{0}\right)$ can be calculated by the following equation due to Brauer:

This completes the proof.

$$
\ell_{p}(G)=\sum_{B \in \mathrm{Blk}^{+}(G, p)} \ell(B)+\left|\operatorname{Irr}^{0}(G)\right| .
$$

TheOREm 5.2. Let $G=\mathrm{J}_{4}$, and let $B$ be a p-block of $G$ with a non-cyclic defect group. Then the number of $B$-weights is the number of irreducible Brauer characters of $B$.

Proof. We may suppose that $p=2$ and $p=3$. If $p=2$ and $B=B_{0}$, then the result follows by Lemma 5.1, Table 1 and (5.1).

Suppose that $p=3$. Since $\operatorname{Irr}\left(N\left(3^{1+2}\right) / C\left(3^{1+2}\right)\right)$ has seven irreducible characters, and since $\operatorname{Irr}\left(N\left(3^{1+2}\right) / 3^{1+2}\right)$ has 14 characters, it follows that $B \in\left\{B_{0}, B_{1}\right\}$ has 7-weights of the form $\left(3^{1+2}, \varphi\right)$. If $\left(3^{2}, b\right)$ is a Brauer $B$-subgroup, then $\operatorname{Irr}^{0}\left(N\left(3^{2}\right) / 3^{2}\right)$ has exactly two characters covering the canonical character of $b$, so $\operatorname{Irr}^{0}\left(N\left(3^{2}\right) / C\left(3^{2}\right)\right)$ has two characters, $B$ has 2-weights of the form $\left(3^{2}, \varphi\right)$, and $B$ has no weight of the form $(3, \varphi)$.

$\operatorname{Irr}^{0}\left(N\left(3^{2}\right) / 3^{2}\right)$ has 9 characters; hence $B_{2}$ has 5-weights of the form $\left(3^{2}, \varphi\right)$.

\section{Radical chains}

Let $G=\mathrm{J}_{4}, C \in \mathcal{R}(G)$ and $N(C)=N_{G}(C)$. We will do some cancellations in the alternating sum of Dade's conjecture. We first list some radical $p$-chains $C(i)$ and their normalizers for certain integers $i$, and then we reduce the proof of the conjecture to the subfamily $\mathcal{R}^{0}(G)$ of $\mathcal{R}(G)$, where $\mathcal{R}^{0}(G)$ is the union of $G$-orbits of all $C(i)$. The subgroups of the 2-chains in Table 3 are given either by Table 1 or in the proof of Lemma 6.1.

Lemma 6.1. Let $\mathcal{R}^{0}(G)$ be the $G$-invariant subfamily of $\mathcal{R}(G)$ such that

$$
\mathcal{R}^{0}(G) / G= \begin{cases}\{C(i): 1 \leqslant i \leqslant 4\}, & \text { with } C(i) \text { given in Table } 2 \text { if } p=3, \\ \{C(i): 1 \leqslant i \leqslant 16\}, & \text { with } C(i) \text { given in Table } 3 \text { if } p=2 .\end{cases}
$$

Then

$$
\sum_{C \in \mathcal{R}(G) / G}(-1)^{|C|} \mathrm{k}\left(N(C), B_{0}, d\right)=\sum_{C \in \mathcal{R}^{0}(G) / G}(-1)^{|C|} \mathrm{k}\left(N(C), B_{0}, d\right)
$$

for all integers $d \geqslant 0$. 
The Alperin weight conjecture and Dade's conjecture for the simple group $\mathrm{J}_{4}$

Table 2: Some radical 3-chains of $\mathrm{J}_{4}$

\begin{tabular}{rrr}
\hline$C$ & & $N(C)$ \\
\hline$C(1)$ & 1 & $\mathrm{~J}_{4}$ \\
$C(2)$ & $1<3$ & $6 \cdot M_{22}: 2$ \\
$C(3)$ & $1<3<3^{2}$ & $\left(3^{2} \times 2^{3}\right) \cdot\left(2 \times S_{3}\right)$ \\
$C(4)$ & $1<3^{2}$ & $\left(3^{2}: 2 \times 2^{3}\right) \cdot S_{4}$ \\
\hline
\end{tabular}

Proof. Let $C \in \mathcal{R}(G)$ be given by (2.1), so that we may suppose that $P_{1} \in \mathcal{R}_{0}(G, p)$.

Case (1). Suppose that $p=3$. Let $C^{\prime}: 1<3<3^{1+2}$ and $g\left(C^{\prime}\right): 1<3^{1+2}$. Then $N\left(C^{\prime}\right)=N\left(g\left(C^{\prime}\right)\right)=N\left(3^{1+2}\right)$,

$$
\mathrm{k}\left(N\left(C^{\prime}\right), B, d\right)=\mathrm{k}\left(N\left(g\left(C^{\prime}\right)\right), B, d\right),
$$

and we may suppose that $C \neq_{G} C^{\prime}$ and $C \neq_{G} g\left(C^{\prime}\right)$. Similarly, let $C^{\prime}: 1<3^{2}<3^{1+2}$ and $g\left(C^{\prime}\right): 1<3<3^{2}<3^{1+2}$. Then $N\left(C^{\prime}\right)=N\left(g\left(C^{\prime}\right)\right)={ }_{G} 3^{1+2} \cdot 2^{3}$, and we may suppose that $C \neq_{G} C^{\prime}$ and $C \neq_{G} g\left(C^{\prime}\right)$. Thus $C={ }_{G} C(i)$ for $1 \leqslant i \leqslant 4$.

Case (2). Suppose that $p=2$. Let $M_{1}=2^{11}: M_{24}, M_{2}=2^{1+12} \cdot\left(3 \cdot M_{22}\right): 2, M_{3}=$ $2^{10}: L_{5}(2)$ and $M_{4}=2^{3+12} \cdot\left(S_{5} \times L_{3}(2)\right)$ be maximal subgroups of $G=\mathrm{J}_{4}$. For each $R \in \mathcal{R}_{0}(G, 2)$, we may suppose that $R \in \mathcal{R}_{0}\left(M_{i}\right)$ such that $N_{G}(R) \leqslant N_{M_{i}}(R)$ for some $i$.

We first classify the radical 2-subgroups of $M_{i}$ using the modified local strategy and do the fusions in $G$ by applying Lemma 2.2. Moreover, we carry out cancellation using Lemma 2.1.

Case (2a). We may take

$$
\begin{aligned}
\mathcal{R}_{0}\left(M_{2}, 2\right)= & \left\{2^{1+12}, 2^{1+12} \cdot 2^{3}, 2^{1+12} \cdot 2^{4}, 2^{10} \cdot 2^{3+4}, 2^{3+12} \cdot 2^{2}, 2^{1+12} \cdot 2^{2+3},\right. \\
& 2^{1+12} \cdot 2^{5}, 2^{1+12} \cdot 2^{3} \cdot 2^{3}, 2^{1+12} \cdot 2^{2} \cdot 2^{4}, 2^{1+12} \cdot 2 \cdot 2^{2+3}, 2^{11} \cdot 2^{2} \cdot 2^{3} \cdot 2^{4}, \\
& \left.2^{1+12} \cdot 2^{2+5}, 2^{1+12} \cdot 2 \cdot 2^{3} \cdot 2^{3}, S\right\},
\end{aligned}
$$

and by [17, Theorem 17], $N(R)=N_{M_{2}}(R)$ for all $R \in \mathcal{R}_{0}\left(M_{2}, 2\right)$, so that we may suppose that $\mathcal{R}_{0}\left(M_{2}, 2\right) \subseteq \mathcal{R}_{0}(G, 2)$.

Let $R \in \mathcal{R}_{0}\left(M_{2}, 2\right) \backslash\left\{2^{1+12}\right\}$ and let $\sigma(R): 1<Q=2^{1+12}<R$, so that $\sigma(R)^{\prime}: 1<R$. Then $\sigma(R)$ and $\sigma(R)^{\prime}$ satisfy the conditions of Lemma 2.1. Thus there is a bijection $g$ from $\mathcal{R}^{-}\left(\sigma(R), 2^{1+12}\right)$ onto $\mathcal{R}^{0}\left(\sigma(R), 2^{1+12}\right)$ such that $N\left(C^{\prime}\right)=N\left(g\left(C^{\prime}\right)\right)$ and $\left|C^{\prime}\right|=$ $\left|g\left(C^{\prime}\right)\right|-1$ for each $C^{\prime} \in \mathcal{R}^{-}\left(\sigma(R), 2^{1+12}\right)$. So (6.2) holds, and we may suppose that

$$
C \notin \bigcup_{R \in \mathcal{R}_{0}\left(M_{2}, 2\right) \backslash\left\{2^{1+12}\right\}}\left(\mathcal{R}^{-}\left(\sigma(R), 2^{1+12}\right) \cup \mathcal{R}^{0}\left(\sigma(R), 2^{1+12}\right)\right) .
$$

In particular, $P_{1} \notin_{G} \mathcal{R}_{0}\left(M_{2}, 2\right) \backslash\left\{2^{1+12}\right\}$ and if $P_{1}=2^{1+12}$, then $C={ }_{G} C(6)$. We may suppose that

$$
\begin{gathered}
P_{1} \in G\left\{2^{10}, 2^{11}, 2^{10} \cdot 2^{4}, 2^{11} \cdot 2^{4}, 2^{3+12}, 2^{3+12} \cdot 2,2^{3} \cdot 2^{6+8}, 2^{2} \cdot 2^{5+10},\right. \\
\left.2^{3+12} \cdot D_{8}, 2^{3+12} \cdot 2^{3}, 2^{11} \cdot 2^{1+6}, 2^{3+12} \cdot 2^{4}, 2^{3+12} \cdot 2^{3} \cdot 2^{2}\right\} .
\end{gathered}
$$


The Alperin weight conjecture and Dade's conjecture for the simple group $\mathrm{J}_{4}$

Table 3: Some radical 2-chains of $\mathrm{J}_{4}$

\begin{tabular}{|c|c|c|}
\hline$C$ & & $N(C)$ \\
\hline$C(1)$ & 1 & $\mathrm{~J}_{4}$ \\
\hline$C(2)$ & $1<2^{11}$ & $2^{11}: M_{24}$ \\
\hline$C(3)$ & $1<2^{11}<2^{1+12} \cdot 2^{4}$ & $2^{1+12} \cdot 2^{4} \cdot 3 \cdot S_{6}$ \\
\hline$C(4)$ & $1<2^{11}<2^{3} \cdot 2^{6+8}<2^{1+12} \cdot 2^{2} \cdot 2^{4}$ & $2^{1+12} \cdot 2^{2} \cdot 2^{4} \cdot\left(S_{3} \times S_{3}\right)$ \\
\hline$C(5)$ & $1<2^{11}<2^{3} \cdot 2^{6+8}$ & $2^{3} \cdot 2^{6+8} \cdot\left(S_{3} \times L_{3}(2)\right)$ \\
\hline$C(6)$ & $1<2^{1+12}$ & $2^{1+12} \cdot 3 \cdot M_{22}: 2$ \\
\hline$C(7)$ & $1<2^{10}<2^{6} .2^{8}$ & $2^{6} \cdot 2^{8} \cdot L_{4}(2)$ \\
\hline$C(8)$ & $1<2^{10}$ & $2^{10}: L_{5}(2)$ \\
\hline$C(9)$ & $1<2^{10}<2^{1+12} \cdot 2^{3}$ & $2^{1+12} \cdot 2^{3}\left(S_{3} \times L_{3}(2)\right)$ \\
\hline$C(10)$ & $1<2^{10}<2^{1+12} \cdot 2^{3}<2^{10} \cdot 2^{2+6}$ & $2^{10} \cdot 2^{2+6} \cdot\left(S_{3} \times S_{3}\right)$ \\
\hline$C(11)$ & $1<2^{10}<2^{3+12} \cdot 2$ & $2^{3+12} \cdot 2 .\left(S_{3} \times L_{3}(2)\right)$ \\
\hline$C(12)$ & $1<2^{10}<2^{3+12} \cdot 2<2^{6} \cdot 2^{8} \cdot 2^{3}$ & $2^{6} \cdot 2^{8} \cdot 2^{3} \cdot L_{3}(2)$ \\
\hline$C(13)$ & $1<2^{10}<2^{3+12} \cdot 2<2^{1+12} \cdot 2^{2+3}<2^{10} \cdot 2^{2} \cdot 2^{3+4}$ & $2^{10} \cdot 2^{2} \cdot 2^{3+4} \cdot S_{3}$ \\
\hline$C(14)$ & $1<2^{10}<2^{3+12} \cdot 2<2^{1+12} \cdot 2^{2+3}$ & $2^{1+12} \cdot 2^{2+3} \cdot\left(S_{3} \times S_{3}\right)$ \\
\hline$C(15)$ & $1<2^{3+12}<2^{3+12} \cdot 2^{2}$ & $2^{3+12} \cdot 2^{2} \cdot\left(S_{3} \times S_{5}\right)$ \\
\hline$C(16)$ & $1<2^{3+12}$ & $2^{3+12} \cdot\left(S_{5} \times L_{3}(2)\right)$ \\
\hline
\end{tabular}

Case (2b). Applying the local strategy $[3,4]$, we obtain sixteen radical subgroups of $M_{4}$. Let

$$
x=\left\{2^{3+12} \cdot 2,2^{3} \cdot 2^{6+8}, 2^{2} \cdot 2^{5+10}, 2^{3+12} \cdot D_{8}, 2^{3+12} \cdot 2^{3}, 2^{3+12} \cdot 2^{4}, 2^{3+12} \cdot 2^{3} \cdot 2^{2}\right\},
$$

so that each subgroup of $\mathcal{X}$ is radical in $G$ and contained in $M_{4}$, by [17]. Using the local structures, we can identify each $R \in \mathcal{X}$ with a radical subgroup of $M_{4}$.

Next we consider fusions of subgroups in $\mathcal{R}_{0}\left(M_{2}, 2\right)$ and $\mathcal{R}_{0}\left(M_{4}, 2\right)$. Let

$$
R=2^{3+12} \cdot 2^{2} \in \mathcal{R}_{0}\left(M_{4}, 2\right),
$$

so that $Z(R)=2$ and $N_{M_{4}}(R)=2^{3+12} \cdot 2^{2} \cdot\left(S_{3} \times S_{5}\right)$. Now $G$ has two classes of involutions $2 A$ and $2 B$ such that $C(2 A)=M_{2}$ and $C(2 B)=2^{11}: M_{22}: 2$. Since $\left|N_{M_{4}}(R)\right|_{2}>$ $|C(2 B)|_{2}$, it follows that $Z(R)$ is a $2 A$ involution. In particular, we may suppose that $N_{M_{4}}(R) \leqslant M_{2}$. By Lemma 2.2 , there is a radical subgroup $P \in \mathcal{R}_{0}\left(M_{2}, 2\right)$ such that $R \leqslant P$ and $N_{M_{4}}(R) \leqslant N_{M_{2}}(P)$. By the local structures of subgroups of $\mathcal{R}_{0}\left(M_{2}, 2\right), R$ is a radical subgroup of $M_{2}$ such that $N_{M_{4}}(R)=N_{M_{2}}(R)=N(R)$, and so is a radical subgroup of $G$. 
Applying the local strategy to $N_{M_{4}}(R)$, we find that $N_{M_{4}}(R)$ has exactly seven radical subgroups $W$, and each is radical in $M_{4}$ with $N_{N_{M_{4}}(R)}(W)=N_{M_{4}}(W)$. In particular,

$$
\mathcal{R}_{0}\left(N_{M_{4}}(R), 2\right)=\mathcal{R}_{0}\left(M_{4}, 2\right) \backslash\left(\mathcal{X} \cup\left\{2^{3+12}\right\}\right) .
$$

If we view $R$ as a subgroup of $\mathcal{R}_{0}\left(M_{2}, 2\right)$ (we can identify $R$ with a radical subgroup of $M_{2}$ using local structures), then each radical subgroup $W$ of $N_{M_{2}}(R)=N_{M_{4}}(R)$ is radical in $M_{2}$ with $N_{M_{2}}(W)=N_{N_{M_{2}}(R)}(W)$. It follows that each $W \in \mathcal{R}_{0}\left(N_{M_{4}}(R), 2\right)$ is radical in $G$ with $N(W)=N_{M_{4}}(W)$, and so each $Q \in \mathcal{R}_{0}\left(M_{4}, 2\right)$ is radical in $G$ with $N(Q)=N_{M_{4}}(Q)$.

We may take

$$
\begin{aligned}
\mathcal{R}_{0}\left(M_{4}, 2\right)= & \left\{2^{3+12}, 2^{3+12} \cdot 2,2^{3+12} \cdot 2^{2}, 2^{3} \cdot 2^{6+8}, 2^{2} \cdot 2^{5+10}, 2^{3+12} \cdot D_{8},\right. \\
& 2^{1+12} \cdot 2^{5}, 2^{1+12} \cdot 2^{2+3}, 2^{3+12} \cdot 2^{3}, 2^{1+12} \cdot 2^{2} \cdot 2^{4}, 2^{3+12} \cdot 2^{4}, \\
& \left.2^{1+12} \cdot 2^{3} \cdot 2^{3}, 2^{1+12} \cdot 2^{2+5}, 2^{11} \cdot 2^{2} \cdot 2^{3} \cdot 2^{4}, 2^{3+12} \cdot 2^{3} \cdot 2^{2}, S\right\}
\end{aligned}
$$

and $N(R)=N_{M_{4}}(R)$ for all $R \in \mathcal{R}_{0}\left(M_{4}, 2\right)$, so that we may suppose that $\mathcal{R}_{0}\left(M_{4}, 2\right) \subseteq$ $\mathcal{R}_{0}(G, 2)$.

Let $R \in \mathcal{X}$ and $\sigma(R): 1<Q=2^{3+12}<R$, so that $\sigma(R)^{\prime}: 1<R$. Then $\sigma(R)$ and $\sigma(R)^{\prime}$ satisfy the conditions of Lemma 2.1. A similar proof to that of Case (2a) shows that we may suppose that

$$
C \notin \bigcup_{R \in \mathcal{X}}\left(\mathcal{R}^{-}\left(\sigma(R), 2^{3+12}\right) \cup \mathcal{R}^{0}\left(\sigma(R), 2^{3+12}\right)\right) .
$$

In particular, $P_{1} \notin_{G} X$, and if $P_{1}=2^{3+12}$, then $P_{2} \notin_{G} X$.

Let $K=2^{3+12} \cdot 2^{2} \cdot\left(S_{3} \times S_{5}\right)$. We may take

$$
\begin{aligned}
\mathcal{R}_{0}(K, 2)= & \left\{2^{3+12} \cdot 2^{2}, 2^{1+12} \cdot 2^{5}, 2^{1+12} \cdot 2^{2+3}, 2^{1+12} \cdot 2^{2} \cdot 2^{4},\right. \\
& \left.2^{1+12} \cdot 2^{3} \cdot 2^{3}, 2^{1+12} \cdot 2^{2+5}, 2^{11} \cdot 2^{2} \cdot 2^{3} \cdot 2^{4}, S\right\} \subseteq \mathcal{R}_{0}\left(M_{4}, 2\right)
\end{aligned}
$$

and $N_{K}(R)=N_{M_{4}}(R)=N(R)$ for all $R \in \mathcal{R}_{0}(K, 2)$, and

$$
\mathcal{R}_{0}(K, 2)=\mathcal{R}_{0}\left(M_{4}, 2\right) \backslash\left(X \cup\left\{2^{3+12}\right\}\right) .
$$

Let $R \in \mathcal{R}_{0}(K, 2) \backslash\left\{2^{3+12} \cdot 2^{2}\right\}$ and let $\sigma(R): 1<2^{3+12}<Q=2^{3+12} \cdot 2^{2}<R$, so that $\sigma(R)^{\prime}: 1<2^{3+12}<R$. A similar proof to that of Case (2a) shows that we may suppose that

$$
C \notin \bigcup_{R \in \mathcal{R}_{0}\left(2^{3+12} \cdot 2^{2} .\left(S_{3} \times S_{5}\right), 2\right)}\left(\mathcal{R}^{-}\left(\sigma(R), 2^{3+12} \cdot 2^{2}\right) \cup \mathcal{R}^{0}\left(\sigma(R), 2^{3+12} \cdot 2^{2}\right)\right) .
$$

In particular, if $P_{1}={ }_{G} 2^{3+12}$, then $C \in_{G}\{C(15), C(16)\}$.

Case (2c). The fusions in $G$ of subgroups of $\mathcal{R}_{0}\left(M_{1}, 2\right)$ with other subgroups in other $\mathcal{R}_{0}\left(M_{i}, 2\right)$ is similar to that of Case $(2 \mathrm{~b})$. We may take

$$
\begin{aligned}
\mathcal{R}_{0}\left(M_{1}, 2\right)=\{ & \left\{2^{11}, 2^{11} \cdot 2^{4}, 2^{1+12} \cdot 2^{4}, 2^{3} \cdot 2^{6+8}, 2^{11} \cdot 2^{1+6}, 2^{3+12} \cdot D_{8},\right. \\
& 2^{1+12} \cdot 2^{2} \cdot 2^{4}, 2^{1+12} \cdot 2 \cdot 2^{2+3}, 2^{3+12} \cdot 2^{4}, 2^{1+12} \cdot 2^{2+5}, \\
& \left.2^{3+12} \cdot 2^{3} \cdot 2^{2}, 2^{11} \cdot 2^{2} \cdot 2^{3} \cdot 2^{4}, 2^{1+12} \cdot 2 \cdot 2^{3} \cdot 2^{3}, S\right\}
\end{aligned}
$$

and $N(R)=N_{M_{1}}(R)$ for all $R \in \mathcal{R}_{0}\left(M_{1}, 2\right)$, so we may suppose that $\mathcal{R}_{0}\left(M_{1}, 2\right) \subseteq$ $\mathcal{R}_{0}(G, 2)$. 
For $R \in\left\{2^{11} \cdot 2^{4}, 2^{11} \cdot 2^{1+6}\right\} \subseteq \mathcal{R}_{0}\left(M_{1}, 2\right)$, let $\sigma(R): 1<Q=2^{11}<R$, so that $\sigma(R)^{\prime}: 1<R$. A similar proof to that of Case (2a) shows that we may suppose that

$$
C \notin \bigcup_{R \in\left\{2^{11} .2^{4}, 2^{11} .2^{1+6}\right\}}\left(\mathcal{R}^{-}\left(\sigma(R), 2^{11}\right) \cup \mathcal{R}^{0}\left(\sigma(R), 2^{11}\right)\right) .
$$

In particular, $P_{1} \neq_{G} 2^{11} .2^{4}$ or $2^{11} .2^{1+6}$, and if $P_{1}=2^{11}$, then $P_{2} \neq_{G} 2^{11} .2^{4}$ and $2^{11} \cdot 2^{1+6}$. Let $L=2^{1+12} \cdot 2^{4} \cdot 3 \cdot S_{6}$. We may take

$$
\begin{aligned}
\mathcal{R}_{0}(L, 2)= & \left\{2^{1+12} \cdot 2^{4}, 2^{1+12} \cdot 2^{2} \cdot 2^{4}, 2^{1+12} \cdot 2 \cdot 2^{2+3},\right. \\
& \left.2^{1+12} \cdot 2^{2+5}, 2^{11} \cdot 2^{2} \cdot 2^{3} \cdot 2^{4}, 2^{1+12} \cdot 2 \cdot 2^{3} \cdot 2^{3}, S\right\} \subseteq \mathcal{R}_{0}\left(M_{1}, 2\right)
\end{aligned}
$$

and $N_{L}(R)=N_{M_{1}}(R)$ for all $R \in \mathcal{R}_{0}(L, 2)$.

Let $R \in \mathcal{R}_{0}(L, 2) \backslash\left\{2^{1+12} \cdot 2^{4}\right\}$, and let $\sigma(R): 1<2^{11}<Q=2^{1+12} \cdot 2^{4}<R$, so that $\sigma(R)^{\prime}: 1<2^{11}<R$. A similar proof to that of Case (2a) shows that we may suppose that

$$
C \notin \bigcup_{R \in \mathcal{R}_{0}\left(2^{1+12} \cdot 2^{4} .3 . S_{6}, 2\right)}\left(\mathcal{R}^{-}\left(\sigma(R), 2^{1+12} \cdot 2^{4}\right) \cup \mathcal{R}^{0}\left(\sigma(R), 2^{1+12} \cdot 2^{4}\right)\right) .
$$

In particular, if $P_{1}=2^{11}$, then $P_{2} \notin_{G} \mathcal{R}_{0}(L, 2) \backslash\left\{2^{1+12} \cdot 2^{4}\right\}$; if, moreover, $P_{2}={ }_{G} 2^{1+12} \cdot 2^{4}$, then $C={ }_{G} C(3)$.

Let $H=2^{3} \cdot 2^{6+8} \cdot\left(S_{3} \times L_{3}(2)\right)$. We may take

$$
\begin{aligned}
\mathcal{R}_{0}(H, 2)= & \left\{2^{3} \cdot 2^{6+8}, 2^{3+12} \cdot D_{8}, 2^{1+12} \cdot 2^{2} \cdot 2^{4}, 2^{3+12} \cdot 2^{4},\right. \\
& \left.2^{1+12} \cdot 2^{2+5}, 2^{3+12} \cdot 2^{3} \cdot 2^{2}, 2^{11} \cdot 2^{2} \cdot 2^{3} \cdot 2^{4}, S\right\} \subseteq \mathcal{R}_{0}\left(M_{1}, 2\right)
\end{aligned}
$$

and $N_{H}(R)=N_{M_{1}}(R)$ for all $R \in \mathcal{R}_{0}(H, 2)$.

Let

$$
y=\left\{2^{3+12} \cdot D_{8}, 2^{3+12} \cdot 2^{4}, 2^{3+12} \cdot 2^{3} \cdot 2^{2}\right\} \subseteq \mathcal{R}_{0}(H, 2), \quad R \in \mathcal{Y},
$$

and let $\sigma(R): 1<2^{11}<Q=2^{3} \cdot 2^{6+8}<R$, so that $\sigma(R)^{\prime}: 1<2^{11}<R$. A similar proof to that of Case (2a) shows that we may suppose that

$$
C \notin \bigcup_{R \in \mathcal{Y}}\left(\mathcal{R}^{-}\left(\sigma(R), 2^{3} \cdot 2^{6+8}\right) \cup \mathcal{R}^{0}\left(\sigma(R), 2^{3} \cdot 2^{6+8}\right)\right) .
$$

In particular, if $P_{1}=2^{11}$, then $P_{2} \notin_{G} \mathcal{Y}$; if, moreover, $P_{2}=2^{3} \cdot 2^{6+8}$, then $P_{3} \notin \mathcal{y}$.

We may take

$$
\mathcal{R}_{0}\left(2^{1+12} \cdot 2^{2} \cdot 2^{4} \cdot\left(S_{3} \times S_{3}\right), 2\right)=\mathcal{R}_{0}(H, 2) \backslash\left(\mathcal{y} \cup\left\{2^{3} \cdot 2^{6+8}\right\} ;\right.
$$

moreover, $N_{2^{1+12} \cdot 2^{2} \cdot 2^{4} .\left(S_{3} \times S_{3}\right)}(R)=N_{H}(R)=N_{M_{1}}(R)$.

For each

$$
R \in \mathcal{R}_{0}\left(2^{1+12} \cdot 2^{2} \cdot 2^{4} \cdot\left(S_{3} \times S_{3}\right), 2\right) \backslash\left\{2^{1+12} \cdot 2^{2} \cdot 2^{4}\right\},
$$

let $\sigma(R): 1<2^{11}<2^{3} \cdot 2^{6+8}<Q=2^{1+12} \cdot 2^{2} \cdot 2^{4}<R$, so that $\sigma(R)^{\prime}: 1<2^{11}<$ $2^{3} .2^{6+8}<R$. A similar proof to that of Case (2a) shows that we may suppose that

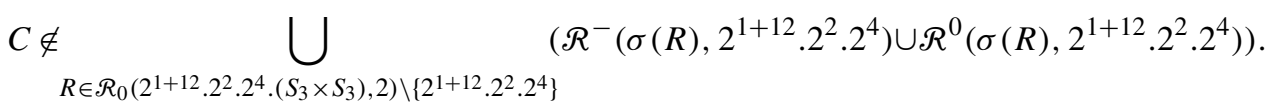

It follows that if $P_{1}=2^{11}$, then $C={ }_{G} C(i)$ for some $2 \leqslant i \leqslant 5$. 
Case (2d). The fusions in $G$ of subgroups of $\mathcal{R}_{0}\left(M_{3}, 2\right)$ with subgroups in other $\mathcal{R}_{0}\left(M_{i}, 2\right)$ is similar to that of Case (2b). We may take

$$
\begin{aligned}
\mathcal{R}_{0}\left(M_{3}, 2\right)=\{ & \left\{2^{10}, 2^{10} \cdot 2^{4}, 2^{6} \cdot 2^{8}, 2^{3+12} \cdot 2,2^{1+12} \cdot 2^{3}, 2^{10} \cdot 2^{3+4}, 2^{6} \cdot 2^{8} \cdot 2^{3},\right. \\
& 2^{10} \cdot 2^{1+6}, 2^{1+12} \cdot 2^{2+3}, 2^{10} \cdot 2^{2+6}, 2^{3+12} \cdot 2^{3}, 2^{1+12} \cdot 2^{3} \cdot 2^{3}, \\
& \left.2^{10} \cdot 2 \cdot 2^{3+5}, 2^{10} \cdot 2^{2} \cdot 2^{3+4}, 2^{10} \cdot 2^{6} \cdot 2^{3}, S\right\}
\end{aligned}
$$

and $N(R)=N_{M_{3}}(R)$ for $R \in \mathcal{R}_{0}\left(M_{3}, 2\right) \backslash \mathcal{Z}$, where

$$
Z=\left\{2^{6} \cdot 2^{8}, 2^{6} \cdot 2^{8} \cdot 2^{3}, 2^{10} \cdot 2^{1+6}, 2^{10} \cdot 2^{2+6}, 2^{10} \cdot 2 \cdot 2^{3+5}, 2^{10} \cdot 2^{2} \cdot 2^{3+4}, 2^{10} \cdot 2^{6} \cdot 2^{3}\right\} \text {. }
$$

In addition, for $R \in \mathcal{Z}, C_{M_{3}}\left(2^{6} .2^{8}\right)=2^{6}, C_{M_{3}}\left(2^{6} \cdot 2^{8} .2^{3}\right) \simeq 2^{3} \simeq C_{M_{3}}\left(2^{10} \cdot 2^{1+6}\right)$,

$$
C_{M_{3}}\left(2^{10} \cdot 2^{2+6}\right) \simeq C_{M_{3}}\left(2^{10} \cdot 2 \cdot 2^{3+5}\right) \simeq C_{M_{3}}\left(2^{10} \cdot 2^{2} \cdot 2^{3+4}\right) \simeq 2,
$$

$C_{M_{3}}\left(2^{10} \cdot 2^{6} \cdot 2^{3}\right) \simeq 2^{2}$ and, moreover,

$$
N_{M_{3}}(R)= \begin{cases}2^{6} \cdot 2^{8} \cdot L_{4}(2), & \text { if } R=2^{6} \cdot 2^{8}, \\ 2^{6} \cdot 2^{8} \cdot 2^{3} \cdot L_{3}(2), & \text { if } R=2^{6} \cdot 2^{8} \cdot 2^{3}, \\ 2^{10} \cdot 2^{1+6} \cdot L_{3}(2), & \text { if } R=2^{10} \cdot 2^{1+6}, \\ 2^{10} \cdot 2^{2+6} \cdot\left(S_{3} \times S_{3}\right), & \text { if } R=2^{10} \cdot 2^{2+6}, \\ 2^{10} \cdot 2 \cdot 2^{3+5} \cdot S_{3}, & \text { if } R=2^{10} \cdot 2 \cdot 2^{3+5}, \\ 2^{10} \cdot 2^{2} \cdot 2^{3+4} \cdot S_{3}, & \text { if } R=2^{10} \cdot 2^{2} \cdot 2^{3+4}, \\ 2^{10} \cdot 2^{6} \cdot 2^{3} \cdot S_{3}, & \text { if } R=2^{10} \cdot 2^{6} \cdot 2^{3} .\end{cases}
$$

Let $\sigma: 1<Q=2^{10}<2^{10} .2^{4}$, so that $\sigma^{\prime}: 1<2^{10} .2^{4}$. A similar proof to that of Case (2a) shows that we may suppose that

$$
C \notin\left(\mathcal{R}^{-}\left(\sigma, 2^{10}\right) \cup \mathcal{R}^{0}\left(\sigma, 2^{10}\right)\right) .
$$

In particular, $P_{1} \neq_{G} 2^{10} .2^{4}$, and if $P_{1}=2^{10}$, then $P_{2} \neq_{G} 2^{10} .2^{4}$.

Let $J=2^{6} \cdot 2^{8} \cdot L_{4}(2)$. We may take

$$
\begin{aligned}
\mathcal{R}_{0}(J, 2)= & \left\{2^{6} \cdot 2^{8}, 2^{6} \cdot 2^{8} \cdot 2^{3}, 2^{10} \cdot 2^{1+6}, 2^{10} \cdot 2^{2+6},\right. \\
& \left.2^{10} \cdot 2 \cdot 2^{3+5}, 2^{10} \cdot 2^{2} \cdot 2^{3+4}, 2^{10} \cdot 2^{6} \cdot 2^{3}, S\right\} \subseteq \mathcal{R}_{0}\left(M_{3}, 2\right)
\end{aligned}
$$

and $N_{J}(R)=N_{M_{3}}(R)$ for all $R \in \mathcal{R}_{0}(J, 2)$.

Let $R \in \mathcal{R}_{0}(J, 2) \backslash\left\{2^{6} .2^{8}\right\}$ and let $\sigma(R): 1<2^{10}<Q=2^{6} .2^{8}<R$, so that $\sigma(R)^{\prime}: 1<2^{10}<R$. A similar proof to that of Case (2a) shows that we may suppose that

$$
C \notin \bigcup_{R \in \mathcal{R}_{0}\left(2^{6} .2^{8} . L_{4}(2), 2\right)}\left(\mathcal{R}^{-}\left(\sigma(R), 2^{6} .2^{8}\right) \cup \mathcal{R}^{0}\left(\sigma(R), 2^{6} .2^{8}\right)\right) .
$$

In particular, if $P_{1}=2^{10}$, then $P_{2} \notin_{G} \mathcal{R}_{0}(J, 2) \backslash\left\{2^{6} .2^{8}\right\}$; if, moreover, $P_{2}={ }_{G} 2^{6} .2^{8}$, then $C={ }_{G} C(7)$.

Let $T=2^{1+12} \cdot 2^{3} .\left(S_{3} \times L_{3}(2)\right)$. We may take

$$
\begin{aligned}
\mathcal{R}_{0}(T, 2)= & \left\{2^{1+12} \cdot 2^{3}, 2^{10} \cdot 2^{3+4}, 2^{1+12} \cdot 2^{2+3}, 2^{10} \cdot 2^{2+6},\right. \\
& \left.2^{1+12} \cdot 2^{3} \cdot 2^{3}, 2^{10} \cdot 2 \cdot 2^{3+5}, 2^{10} \cdot 2^{2} \cdot 2^{3+4}, S\right\} \subseteq \mathcal{R}_{0}\left(M_{3}, 2\right)
\end{aligned}
$$

and $N_{T}(R)=N_{M_{3}}(R)$ for all $R \in \mathcal{R}_{0}(T, 2)$.

Let

$$
\mathcal{T}=\left\{2^{10} \cdot 2^{3+4}, 2^{1+12} \cdot 2^{2+3}, 2^{1+12} \cdot 2^{3} \cdot 2^{3}\right\} \subseteq \mathcal{R}_{0}(T, 2)
$$


The Alperin weight conjecture and Dade's conjecture for the simple group $\mathrm{J}_{4}$

$R \in \mathcal{T}$, and let $\sigma(R): 1<2^{10}<Q=2^{1+12} \cdot 2^{3}<R$, so that $\sigma(R)^{\prime}: 1<2^{10}<R$. A similar proof to that of Case (2a) shows that we may suppose that

$$
C \notin \bigcup_{R \in \mathcal{T}}\left(\mathcal{R}^{-}\left(\sigma(R), 2^{1+12} \cdot 2^{3}\right) \cup \mathcal{R}^{0}\left(\sigma(R), 2^{1+12} \cdot 2^{3}\right)\right) .
$$

In particular, if $P_{1}=2^{10}$, then $P_{2} \notin_{G} \mathcal{T}$; if, moreover, $P_{2}=2^{1+12} .2^{3}$, then $P_{3} \notin \mathcal{T}$.

Let $V=2^{3+12} \cdot 2 .\left(S_{3} \times L_{3}(2)\right)$. We may take

$$
\begin{aligned}
\mathcal{R}_{0}(V, 2)= & \left\{2^{3+12} \cdot 2,2^{6} \cdot 2^{8} \cdot 2^{3}, 2^{1+12} \cdot 2^{2+3}, 2^{3+12} \cdot 2^{3},\right. \\
& \left.2^{1+12} \cdot 2^{3} \cdot 2^{3}, 2^{10} \cdot 2^{2} \cdot 2^{3+4}, 2^{10} \cdot 2^{6} \cdot 2^{3}, S\right\} \subseteq \mathcal{R}_{0}\left(M_{3}, 2\right)
\end{aligned}
$$

and $N_{V}(R)=N_{M_{3}}(R)$ for all $R \in \mathcal{R}_{0}(V, 2)$.

Let $\sigma: 1<2^{10}<Q=2^{3+12} \cdot 2<2^{3+12} \cdot 2^{3}$, so that $\sigma(R)^{\prime}: 1<2^{10}<R$. A similar proof to that of Case (2a) shows that we may suppose that

$$
C \notin\left(\mathcal{R}^{-}\left(\sigma, 2^{3+12} .2\right) \cup \mathcal{R}^{0}\left(\sigma, 2^{3+12} .2\right)\right) \text {. }
$$

In particular, if $P_{1}=2^{10}$, then $P_{2} \notin_{G} 2^{3+12} \cdot 2^{3}$ and if moreover, $P_{2}=2^{3+12} \cdot 2$, then $P_{3} \neq 2^{3+12} \cdot 2^{3}$.

We may take

$$
\mathcal{R}_{0}\left(2^{10} \cdot 2^{2+6} \cdot\left(S_{3} \times S_{3}\right), 2\right)=\left\{2^{10} \cdot 2^{2+6}, 2^{10} \cdot 2 \cdot 2^{3+5}, 2^{10} \cdot 2^{2} \cdot 2^{3+4}, S\right\} \subseteq \mathcal{R}_{0}\left(M_{3}, 2\right) ;
$$

moreover, $N_{2^{10} .2^{2+6} .\left(S_{3} \times S_{3}\right)}(R)=N_{M_{3}}(R)$.

For each

$$
R \in \mathcal{R}_{0}\left(2^{10} \cdot 2^{2+6} .\left(S_{3} \times S_{3}\right), 2\right) \backslash\left\{2^{10} .2^{2+6}\right\},
$$

let

$$
\sigma(R): 1<2^{10}<2^{1+12} \cdot 2^{3}<Q=2^{10} \cdot 2^{2+6}<R,
$$

so that

$$
\sigma(R)^{\prime}: 1<2^{10}<2^{1+12} \cdot 2^{3}<R .
$$

A similar proof to that of Case (2a) shows that we may suppose that

$$
C \notin \underset{R \in \mathcal{R}_{0}\left(2^{10} .2^{2+6} .\left(S_{3} \times S_{3}\right), 2\right) \backslash\left\{2^{10} .2^{2+6}\right\}}{\bigcup^{-}}\left(\mathcal{R}^{-}\left(\sigma(R), 2^{10} \cdot 2^{2+6}\right) \cup \mathcal{R}^{0}\left(\sigma(R), 2^{10} .2^{2+6}\right)\right) \text {. }
$$

It follows that if $P_{1}=2^{10}$ and $P_{2}=2^{1+12} \cdot 2^{3}$, then $C \in\{C(9), C(10)\}$.

We may take

$$
\mathcal{R}_{0}\left(2^{6} \cdot 2^{8} \cdot 2^{3} \cdot L_{3}(2), 2\right)=\left\{2^{6} \cdot 2^{8} \cdot 2^{3}, 2^{10} \cdot 2^{2} \cdot 2^{3+4}, 2^{10} \cdot 2^{6} \cdot 2^{3}, S\right\} \subseteq \mathcal{R}_{0}\left(M_{3}, 2\right) ;
$$

moreover, $N_{2^{6} \cdot 2^{8} \cdot 2^{3} \cdot L_{3}(2)}(R)=N_{M_{3}}(R)$.

For each

$$
R \in \mathcal{R}_{0}\left(2^{6} \cdot 2^{8} \cdot 2^{3} \cdot L_{3}(2), 2\right) \backslash\left\{2^{6} \cdot 2^{8} \cdot 2^{3}\right\}
$$

let

$$
\sigma(R): 1<2^{10}<2^{3+12} \cdot 2<Q=2^{6} \cdot 2^{8} \cdot 2^{3}<R,
$$

so that $\sigma(R)^{\prime}: 1<2^{10}<2^{3+12} .2<R$. A similar proof to that of Case (2a) shows that we may suppose that

$$
C \notin \underset{R \in \mathcal{R}_{0}\left(2^{6} \cdot 2^{8} \cdot 2^{3} \cdot L_{3}(2), 2\right) \backslash\left\{2^{6} \cdot 2^{8} \cdot 2^{3}\right\}}{\bigcup^{-}}\left(\mathcal{R}^{-}\left(\sigma(R), 2^{6} \cdot 2^{8} \cdot 2^{3}\right) \cup \mathcal{R}^{0}\left(\sigma(R), 2^{6} \cdot 2^{8} \cdot 2^{3}\right)\right) .
$$


So if $P_{1}=2^{10}$ and $P_{2}=2^{3+12} \cdot 2$, then $P_{3} \notin_{G} \mathcal{R}_{0}\left(2^{6} \cdot 2^{8} \cdot 2^{3} \cdot L_{3}(2), 2\right) \backslash\left\{2^{6} \cdot 2^{8} \cdot 2^{3}\right\}$, and if moreover, $P_{3}=2^{6} \cdot 2^{8} \cdot 2^{3}$, then $C={ }_{G} C(11)$.

We may take

$\mathcal{R}_{0}\left(2^{1+12} \cdot 2^{2+3} \cdot\left(S_{3} \times S_{3}\right), 2\right)=\left\{2^{1+12} \cdot 2^{2+3}, 2^{1+12} \cdot 2^{3} \cdot 2^{3}, 2^{10} \cdot 2^{2} \cdot 2^{3+4}, S\right\} \subseteq \mathcal{R}_{0}\left(M_{3}, 2\right)$ and, moreover, $N_{2^{1+12} \cdot 2^{2+3} \cdot\left(S_{3} \times S_{3}\right)}(R)=N_{M_{3}}(R)$.

Let

$$
\sigma: 1<2^{10}<2^{3+12} \cdot 2<Q=2^{1+12} \cdot 2^{2+3}<2^{1+12} \cdot 2^{3} \cdot 2^{3}
$$

so that

$$
\sigma^{\prime}: 1<2^{10}<2^{3+12} \cdot 2<2^{1+12} \cdot 2^{3} \cdot 2^{3} .
$$

A similar proof to that of Case (2a) shows that we may suppose that

$$
C \notin\left(\mathcal{R}^{-}\left(\sigma, 2^{1+12} \cdot 2^{2+3}\right) \cup \mathcal{R}^{0}\left(\sigma, 2^{1+12} \cdot 2^{2+3}\right)\right) .
$$

It follows that if $P_{1}=2^{10}$ and $P_{2}=2^{3+12} \cdot 2$, then $P_{3} \neq_{G} 2^{1+12} \cdot 2^{3} \cdot 2^{3}$ and if, moreover, $P_{3}=2^{1+12} \cdot 2^{2+3}$, then $P_{4} \neq \neq_{G} 2^{1+12} \cdot 2^{3} \cdot 2^{3}$.

Let

$$
\sigma: 1<2^{10}<2^{3+12} \cdot 2<2^{1+12} \cdot 2^{2+3}<Q=2^{10} \cdot 2^{2} \cdot 2^{3+4}<S,
$$

so that

$$
\sigma^{\prime}: 1<2^{10}<2^{3+12} \cdot 2<2^{1+12} \cdot 2^{2+3}<S .
$$

A similar proof to that of Case (2a) shows that we may suppose that

$$
C \notin\left(\mathcal{R}^{-}\left(\sigma, 2^{10} \cdot 2^{2} \cdot 2^{3+4}\right) \cup \mathcal{R}^{0}\left(\sigma, 2^{10} \cdot 2^{2} \cdot 2^{3+4}\right)\right) .
$$

It follows that if $P_{1}=2^{10}$, then $C \in\{C(i): 7 \leqslant i \leqslant 14\}$.

\section{The proof of Dade's ordinary conjecture}

Let $L=N(C)$ be the normalizer of a radical $p$-chain. If $L$ is a maximal subgroup of $\mathrm{J}_{4}$, then the character table of $L$ can be found in the library of character tables distributed with GAP. If $L$ is not a maximal subgroup, its character table can be calculated easily using MAGMA.

The tables listing the degrees of irreducible characters referenced in the proof of Theorem 7.1 are given in Appendix A.

THeOREM 7.1. Let $B$ be a p-block of $G=\mathrm{J}_{4}$ with a positive defect. Then B satisfies Dade's ordinary conjecture.

Proof. By [11] and [9], we may suppose that $p=2$ or $p=3$, and a defect group of $B$ is non-cyclic. By Lemma 5.1, $B \in\left\{B_{0}, B_{1}, B_{2}\right\}$ when $p=3$ and $B=B_{0}$ when $p=2$.

Case (1). Suppose that $p=3$. If $B=B_{2}$, then

$$
\mathrm{k}\left(N(C(1)), B_{2}, d\right)=\mathrm{k}\left(N(C(4)), B_{2}, d\right)= \begin{cases}9, & \text { if } d=2, \\ 0, & \text { otherwise }\end{cases}
$$

and

$$
\mathrm{k}\left(N(C(3)), B_{2}, d\right)=\mathrm{k}\left(N(C(2)), B_{2}, d\right)= \begin{cases}18, & \text { if } d=2, \\ 0, & \text { otherwise }\end{cases}
$$


The Alperin weight conjecture and Dade's conjecture for the simple group $\mathrm{J}_{4}$

Table 4: Values of $\mathrm{k}(i, d, u)$ when $p=2$ and $\mathrm{d}(N(C(i)))=20$

\begin{tabular}{l|rrrrrrrrrrrc}
\hline Defect d & 20 & 19 & 18 & 17 & 16 & 15 & 14 & 13 & 12 & 11 & 10 & otherwise \\
\hline $\mathrm{k}(7, \mathrm{~d}, \mathrm{u})$ & 32 & 16 & 36 & 28 & 2 & 2 & 10 & 2 & 0 & 0 & 1 & 0 \\
$\mathrm{k}(8, \mathrm{~d}, \mathrm{u})$ & 32 & 16 & 20 & 8 & 2 & 0 & 2 & 0 & 0 & 0 & 1 & 0 \\
$\mathrm{k}(9, \mathrm{~d}, \mathrm{u})$ & 32 & 32 & 52 & 24 & 18 & 8 & 18 & 12 & 2 & 2 & 1 & 0 \\
$\mathrm{k}(10, \mathrm{~d}, \mathrm{u})$ & 32 & 32 & 84 & 100 & 26 & 26 & 26 & 14 & 6 & 4 & 1 & 0 \\
$\mathrm{k}(11, \mathrm{~d}, \mathrm{u})$ & 32 & 56 & 28 & 16 & 38 & 32 & 6 & 0 & 0 & 0 & 0 & 0 \\
$\mathrm{k}(12, \mathrm{~d}, \mathrm{u})$ & 32 & 56 & 44 & 36 & 38 & 34 & 14 & 2 & 0 & 0 & 0 & 0 \\
$\mathrm{k}(13, \mathrm{~d}, \mathrm{u})$ & 32 & 72 & 92 & 124 & 62 & 58 & 30 & 22 & 10 & 2 & 0 & 0 \\
$\mathrm{k}(14, \mathrm{~d}, \mathrm{u})$ & 32 & 72 & 60 & 48 & 54 & 40 & 22 & 20 & 6 & 0 & 0 & 0 \\
\hline
\end{tabular}

This proves the theorem when $B=B_{2}$.

If $B=B_{0}$ or $B_{1}$, then

$$
\mathrm{k}(N(C(1)), B, d)=\mathrm{k}(N(C(2)), B, d)= \begin{cases}9, & \text { if } d=3, \\ 5, & \text { if } d=2, \\ 0, & \text { otherwise }\end{cases}
$$

and

$$
\mathrm{k}(N(C(3)), B, d)=\mathrm{k}(N(C(4)), B, d)= \begin{cases}9, & \text { if } d=3, \\ 2, & \text { if } d=2, \\ 0, & \text { otherwise }\end{cases}
$$

This proves the theorem when $p=3$.

Case (2). Suppose that $p=2$, so that $B=B_{0}$. We set $\mathrm{k}(i, d, u)=\mathrm{k}(N(C(i)), B, d, u)$ for integers $i, d$ and $u$.

We first consider the chains $C$ with $\mathrm{d}(N(C))=20$, so that $C={ }_{G} C(i)$ for $7 \leqslant i \leqslant 14$. The values $\mathrm{k}(i, d, u)$ are given in Table 4 .

It follows that

$$
\sum_{i=7}^{14}(-1)^{|C(i)|} \mathrm{k}\left(N(C(i)), B_{0}, d\right)=0 .
$$

Finally, suppose that $C=C(i)$ is a chain with $\mathrm{d}(N(C))=21$. Then $C={ }_{G} C(i)$ for $1 \leqslant i \leqslant 6$ or $15 \leqslant i \leqslant 16$. The values $\mathrm{k}(i, d, u)$ are given in Table 5 .

It follows that

$$
\sum_{\mathrm{d}(N(C))=21}(-1)^{|C|} \mathrm{k}\left(N(C), B_{0}, d, u\right)=0,
$$

and Theorem 7.1 follows. 
The Alperin weight conjecture and Dade's conjecture for the simple group $\mathrm{J}_{4}$

Table 5: Values of $\mathrm{k}(i, d, u)$ when $p=2$ and $\mathrm{d}(N(C(i)))=21$

\begin{tabular}{l|rrrrrrrrrrrrc}
\hline Defect d & 21 & 20 & 19 & 18 & 17 & 16 & 15 & 14 & 13 & 12 & 11 & 7 & otherwise \\
\hline $\mathrm{k}(1, \mathrm{~d}, \mathrm{u})$ & 32 & 8 & 4 & 4 & 1 & 0 & 4 & 4 & 1 & 0 & 0 & 1 & 0 \\
$\mathrm{k}(2, \mathrm{~d}, \mathrm{u})$ & 32 & 8 & 4 & 12 & 7 & 0 & 4 & 4 & 1 & 0 & 0 & 0 & 0 \\
$\mathrm{k}(3, \mathrm{~d}, \mathrm{u})$ & 32 & 24 & 12 & 28 & 11 & 12 & 11 & 12 & 11 & 6 & 1 & 0 & 0 \\
$\mathrm{k}(4, \mathrm{~d}, \mathrm{u})$ & 32 & 40 & 28 & 40 & 21 & 30 & 27 & 14 & 15 & 4 & 1 & 0 & 0 \\
$\mathrm{k}(5, \mathrm{~d}, \mathrm{u})$ & 32 & 24 & 20 & 24 & 9 & 18 & 20 & 6 & 1 & 0 & 0 & 0 & 0 \\
$\mathrm{k}(6, \mathrm{~d}, \mathrm{u})$ & 32 & 24 & 12 & 4 & 1 & 4 & 5 & 12 & 11 & 6 & 1 & 1 & 0 \\
$\mathrm{k}(15, \mathrm{~d}, \mathrm{u})$ & 32 & 40 & 28 & 16 & 11 & 22 & 21 & 14 & 15 & 4 & 1 & 0 & 0 \\
$\mathrm{k}(16, \mathrm{~d}, \mathrm{u})$ & 32 & 24 & 20 & 16 & 3 & 18 & 20 & 6 & 1 & 0 & 0 & 0 & 0 \\
\hline
\end{tabular}

Appendix A. Degrees of character tables for chain normalisers of $\mathbf{J}_{4}$

Table A.1: The degrees of characters in $\operatorname{Irr}\left(\mathrm{J}_{4}\right)$

\begin{tabular}{|c|c|c|c|c|c|}
\hline Degree: & 1 & 1333 & 299367 & 887778 & 889111 \\
\hline Number: & 1 & 2 & 2 & 2 & 1 \\
\hline Degree: & 1187145 & 1776888 & 3403149 & 4290927 & 32307363 \\
\hline Number: & 2 & 1 & 2 & 1 & 2 \\
\hline Degree: & 32897107 & 35411145 & 95288172 & 230279749 & 259775040 \\
\hline Number: & 2 & 2 & 1 & 1 & 2 \\
\hline Degree: & 300364890 & 366159104 & 393877506 & 394765284 & 460559498 \\
\hline Number: & 1 & 1 & 1 & 1 & 1 \\
\hline Degree: & 493456605 & 690839247 & 786127419 & 789530568 & 885257856 \\
\hline Number: & 1 & 1 & 3 & 1 & 2 \\
\hline Degree: & 1016407168 & 1085604531 & 1089007680 & 1182518964 & 1183406741 \\
\hline Number: & 2 & 1 & 1 & 1 & 2 \\
\hline Degree: & 1184295852 & 1445942610 & 1509863773 & 1579061136 & 1842237992 \\
\hline Number: & 1 & 3 & 1 & 1 & 1 \\
\hline Degree: & 1903741279 & 1981808640 & 2001151845 & 2267824128 & 2692972480 \\
\hline Number: & 1 & 3 & 3 & 1 & 1 \\
\hline $\begin{array}{l}\text { Degree: } \\
\text { Numbr. }\end{array}$ & 2727495848 & 3054840657 & & & \\
\hline & & & & & \\
\hline
\end{tabular}


The Alperin weight conjecture and Dade's conjecture for the simple group $\mathrm{J}_{4}$

Table A.2: The degrees of characters in $\operatorname{Irr}\left(2^{11}: M_{24}\right)$

\begin{tabular}{r|rrrrrrr}
\hline Degree: & 1 & 23 & 45 & 231 & 252 & 253 & 483 \\
Number: & 1 & 1 & 2 & 2 & 1 & 1 & 1 \\
\hline Degree: & 759 & 770 & 990 & 1035 & 1265 & 1288 & 1771 \\
Number: & 1 & 2 & 2 & 3 & 1 & 2 & 1 \\
\hline Degree: & 2024 & 2277 & 3312 & 3520 & 5313 & 5544 & 5796 \\
Number: & 1 & 1 & 1 & 1 & 2 & 1 & 1 \\
\hline Degree: & 10395 & 10626 & 11385 & 15180 & 15939 & 21252 & 26565 \\
Number: & 1 & 1 & 1 & 1 & 3 & 1 & 1 \\
\hline Degree: & 28336 & 34155 & 41216 & 42504 & 48576 & 53130 & 57960 \\
Number: & 1 & 4 & 1 & 1 & 1 & 1 & 2 \\
\hline Degree: & 68310 & 69552 & 70840 & 79695 & 85008 & 91080 & 127512 \\
Number: & 1 & 2 & 2 & 3 & 2 & 1 & 2 \\
\hline Degree: & 141680 & 154560 & 159390 & 185472 & 226688 & 239085 & \\
Number: & 1 & 2 & 1 & 2 & 2 & 2 & \\
\hline
\end{tabular}

Table A.3: The degrees of characters in $\operatorname{Irr}\left(2^{1+12} \cdot 2^{4} \cdot 3 \cdot S_{6}\right)$

\begin{tabular}{r|rrrrrrrrrr}
\hline Degree: & 1 & 5 & 6 & 9 & 10 & 12 & 15 & 16 & 18 & 30 \\
Number: & 2 & 4 & 2 & 2 & 2 & 1 & 4 & 1 & 3 & 5 \\
\hline Degree: & 45 & 60 & 72 & 90 & 108 & 135 & 180 & 270 & 288 & 360 \\
Number: & 8 & 1 & 2 & 8 & 1 & 12 & 5 & 4 & 4 & 10 \\
\hline Degree: & 384 & 540 & 576 & 640 & 720 & 768 & 1080 & 1152 & 1280 & 1440 \\
Number: & 2 & 4 & 1 & 4 & 4 & 1 & 16 & 4 & 1 & 8 \\
\hline Degree: & 1536 & 1728 & 1920 & 2160 & 2304 & 2560 & 2880 & 3072 & 3840 & \\
Number: & 2 & 4 & 2 & 6 & 4 & 4 & 6 & 1 & 5 & \\
\hline
\end{tabular}

Table A.4: The degrees of characters in $\operatorname{Irr}\left(2^{1+12} \cdot 2^{2} \cdot 2^{4} \cdot\left(S_{3} \times S_{3}\right)\right.$

\begin{tabular}{r|rrrrrrrrrrr}
\hline Degree & 1 & 2 & 3 & 4 & 6 & 9 & 12 & 18 & 24 & 36 & 48 \\
Number & 4 & 4 & 16 & 1 & 10 & 12 & 5 & 26 & 4 & 22 & 1 \\
\hline Degree & 72 & 96 & 144 & 192 & 256 & 288 & 384 & 512 & 576 & 768 & 1024 \\
Number & 36 & 4 & 20 & 13 & 4 & 26 & 14 & 4 & 14 & 11 & 1 \\
\hline
\end{tabular}


The Alperin weight conjecture and Dade's conjecture for the simple group $\mathrm{J}_{4}$

Table A.5: The degrees of characters in $\operatorname{Irr}\left(2^{3} .2^{6+8} \cdot\left(L_{3}(2) \times S_{3}\right)\right)$

\begin{tabular}{r|rrrrrrrrrr}
\hline Degree: & 1 & 2 & 3 & 6 & 7 & 8 & 9 & 12 & 14 & 16 \\
Number: & 2 & 1 & 6 & 4 & 2 & 2 & 4 & 1 & 1 & 1 \\
\hline Degree: & 18 & 21 & 24 & 42 & 63 & 84 & 126 & 168 & 252 & 336 \\
Number: & 2 & 10 & 2 & 8 & 8 & 6 & 8 & 6 & 13 & 5 \\
\hline Degree: & 448 & 504 & 672 & 896 & 1008 & 1344 & 1792 & 2016 & 2688 & 4032 \\
Number: & 4 & 14 & 8 & 4 & 3 & 14 & 1 & 10 & 2 & 2 \\
\hline
\end{tabular}

Table A.6: The degrees of characters in $\operatorname{Irr}\left(2^{1+12} \cdot 3 \cdot M_{22}: 2\right)$

\begin{tabular}{r|rrrrrrrr}
\hline Degree: & 1 & 21 & 42 & 45 & 55 & 90 & 99 & 154 \\
Number: & 2 & 2 & 1 & 4 & 2 & 2 & 2 & 2 \\
\hline Degree: & 198 & 210 & 231 & 385 & 420 & 462 & 560 & 640 \\
Number: & 1 & 4 & 2 & 2 & 1 & 2 & 1 & 4 \\
\hline Degree: & 660 & 693 & 768 & 1386 & 2016 & 2772 & 3465 & 3584 \\
Number: & 1 & 4 & 1 & 2 & 2 & 4 & 4 & 2 \\
\hline Degree: & 4158 & 5544 & 6930 & 7680 & 8316 & 8448 & 10395 & 13440 \\
Number: & 2 & 2 & 4 & 2 & 1 & 2 & 8 & 2 \\
\hline Degree: & 13860 & 15360 & 16128 & 19712 & 20160 & 20790 & 21120 & 22176 \\
Number: & 5 & 1 & 3 & 1 & 5 & 4 & 2 & 2 \\
\hline Degree: & 24192 & 26880 & 27720 & 28160 & 42240 & 49152 & & \\
Number: & 4 & 3 & 2 & 2 & 1 & 1 & & \\
\hline
\end{tabular}

Table A.7: The degrees of characters in $\operatorname{Irr}\left(2^{6} \cdot 2^{8} \cdot L_{4}(2)\right)$

\begin{tabular}{r|rrrrrrrrrrr}
\hline Degree: & 1 & 7 & 14 & 15 & 20 & 21 & 28 & 35 & 45 & 56 & 64 \\
Number: & 1 & 1 & 1 & 3 & 1 & 3 & 1 & 1 & 8 & 1 & 1 \\
\hline Degree: & 70 & 90 & 105 & 120 & 140 & 210 & 280 & 315 & 420 & 448 & 560 \\
Number: & 1 & 3 & 9 & 3 & 4 & 5 & 4 & 6 & 13 & 2 & 1 \\
\hline Degree: & 630 & 840 & 1260 & 1680 & 2240 & 2520 & 3360 & 4032 & 4480 & 6720 & 7168 \\
Number: & 6 & 8 & 17 & 1 & 4 & 12 & 2 & 2 & 2 & 1 & 1 \\
\hline
\end{tabular}


The Alperin weight conjecture and Dade's conjecture for the simple group $\mathrm{J}_{4}$

Table A.8: The degrees of characters in $\operatorname{Irr}\left(2^{10}: L_{5}(2)\right)$

\begin{tabular}{r|rrrrrrrr}
\hline Degree: & 1 & 30 & 124 & 155 & 217 & 280 & 310 & 315 \\
Number: & 1 & 1 & 1 & 3 & 1 & 1 & 1 & 6 \\
\hline Degree: & 465 & 496 & 651 & 868 & 930 & 960 & 1024 & 1085 \\
Number: & 8 & 1 & 3 & 3 & 7 & 1 & 1 & 2 \\
\hline Degree: & 1240 & 1860 & 2170 & 2480 & 3255 & 4340 & 6510 & 7812 \\
Number: & 3 & 1 & 1 & 1 & 4 & 4 & 4 & 2 \\
\hline Degree: & 8680 & 9765 & 13020 & 13888 & 19530 & 26040 & 39060 & \\
Number: & 2 & 4 & 5 & 1 & 2 & 2 & 4 & \\
\hline
\end{tabular}

Table A.9: The degrees of characters in $\operatorname{Irr}\left(2^{1+12} \cdot 2^{3} \cdot\left(S_{3} \times L_{3}(2)\right)\right)$

\begin{tabular}{r|rrrrrrrrr}
\hline Degree: & 1 & 2 & 3 & 6 & 7 & 8 & 12 & 14 & 16 \\
Number: & 2 & 1 & 4 & 4 & 6 & 2 & 1 & 5 & 1 \\
\hline Degree: & 21 & 28 & 42 & 63 & 64 & 84 & 126 & 128 & 168 \\
Number: & 12 & 1 & 8 & 8 & 2 & 17 & 14 & 1 & 10 \\
\hline Degree: & 192 & 252 & 336 & 384 & 448 & 504 & 512 & 672 & 768 \\
Number: & 4 & 33 & 5 & 4 & 6 & 12 & 2 & 6 & 1 \\
\hline Degree: & 896 & 1008 & 1024 & 1344 & 1792 & 2016 & 2688 & & \\
Number: & 5 & 12 & 1 & 6 & 1 & 2 & 2 & & \\
\hline
\end{tabular}

Table A.10: The degrees of characters in $\operatorname{Irr}\left(2^{10} \cdot 2^{2+6} \cdot\left(S_{3} \times S_{3}\right)\right)$

\begin{tabular}{r|rrrrrrrrrrrrr}
\hline Degree: & 1 & 2 & 3 & 4 & 6 & 8 & 9 & 12 & 16 & 18 & 24 & 36 & 48 \\
Number: & 4 & 4 & 12 & 5 & 10 & 4 & 16 & 26 & 1 & 18 & 14 & 53 & 1 \\
\hline Degree: & 64 & 72 & 96 & 128 & 144 & 192 & 256 & 288 & 384 & 512 & 576 & 768 & 1024 \\
Number: & 4 & 82 & 4 & 4 & 24 & 18 & 5 & 22 & 10 & 4 & 4 & 1 & 1 \\
\hline
\end{tabular}

Table A.11: The degrees of characters in $\operatorname{Irr}\left(2^{3+12} .2 .\left(S_{3} \times L_{3}(2)\right)\right.$

\begin{tabular}{r|rrrrrrrrr}
\hline Degree: & 1 & 2 & 3 & 6 & 7 & 8 & 12 & 14 & 16 \\
Number: & 4 & 2 & 8 & 8 & 4 & 4 & 2 & 2 & 2 \\
\hline Degree: & 21 & 42 & 63 & 84 & 112 & 126 & 168 & 224 & 252 \\
Number: & 8 & 16 & 8 & 10 & 8 & 28 & 2 & 8 & 16 \\
\hline Degree: & 336 & 448 & 504 & 672 & 1008 & 1344 & 2016 & & \\
Number: & 16 & 2 & 10 & 16 & 12 & 4 & 8 & & \\
\hline
\end{tabular}


The Alperin weight conjecture and Dade's conjecture for the simple group $\mathrm{J}_{4}$

Table A.12: The degrees of characters in $\operatorname{Irr}\left(2^{6} \cdot 2^{8} \cdot 2^{3} \cdot L_{3}(2)\right)$

\begin{tabular}{r|rrrrrrrrrr}
\hline Degree: & 1 & 3 & 6 & 7 & 8 & 14 & 21 & 28 & 42 & 56 \\
Number: & 4 & 8 & 4 & 12 & 4 & 16 & 8 & 10 & 36 & 2 \\
\hline Degree: & 84 & 112 & 168 & 224 & 336 & 448 & 672 & 896 & 1344 & \\
Number: & 34 & 16 & 30 & 20 & 22 & 10 & 14 & 2 & 4 & \\
\hline
\end{tabular}

Table A.13: The degrees of characters in $\operatorname{Irr}\left(2^{10} \cdot 2^{2} \cdot 2^{3+4} \cdot S_{3}\right)$

\begin{tabular}{r|rrrrrrrrrrrrrrrr}
\hline Degree: & 1 & 2 & 3 & 4 & 6 & 8 & 12 & 24 & 48 & 64 & 96 & 128 & 192 & 256 & 384 & 512 \\
Number: & 16 & 20 & 16 & 10 & 52 & 2 & 82 & 122 & 62 & 16 & 58 & 20 & 14 & 10 & 2 & 2 \\
\hline
\end{tabular}

Table A.14: The degrees of characters in $\operatorname{Irr}\left(2^{1+12} \cdot 2^{2+3} \cdot\left(S_{3} \times S_{3}\right)\right)$

\begin{tabular}{r|rrrrrrrrrrr}
\hline Degree: & 1 & 2 & 3 & 4 & 6 & 9 & 12 & 18 & 24 & 36 & 48 \\
Number: & 8 & 8 & 16 & 2 & 16 & 8 & 4 & 48 & 4 & 54 & 22 \\
\hline Degree: & 64 & 72 & 96 & 128 & 144 & 192 & 256 & 288 & 384 & 576 & 768 \\
Number: & 8 & 44 & 14 & 8 & 32 & 10 & 2 & 26 & 12 & 4 & 4 \\
\hline
\end{tabular}

Table A.15: The degrees of characters in $\operatorname{Irr}\left(2^{3+12} \cdot 2^{2} \cdot\left(S_{3} \times S_{5}\right)\right)$

\begin{tabular}{r|rrrrrrrrrrr}
\hline Degree: & 1 & 2 & 3 & 4 & 5 & 6 & 8 & 10 & 12 & 15 & 18 \\
Number: & 4 & 2 & 4 & 4 & 4 & 2 & 2 & 2 & 5 & 12 & 2 \\
\hline Degree: & 30 & 45 & 60 & 90 & 120 & 180 & 240 & 360 & 480 & 576 & 640 \\
Number: & 6 & 8 & 1 & 26 & 2 & 18 & 5 & 12 & 20 & 8 & 8 \\
\hline Degree: & 720 & 768 & 960 & 1152 & 1280 & 1440 & 1536 & 1920 & 2304 & 2560 & 3072 \\
Number: & 6 & 6 & 13 & 4 & 8 & 2 & 2 & 2 & 1 & 2 & 1 \\
\hline
\end{tabular}

Table A.16: The degrees of characters in $\operatorname{Irr}\left(2^{3+12} \cdot\left(S_{5} \times L_{3}(2)\right)\right)$

\begin{tabular}{r|rrrrrrrrrr}
\hline Degree: & 1 & 3 & 4 & 5 & 6 & 7 & 8 & 12 & 15 & 18 \\
Number: & 2 & 4 & 2 & 2 & 3 & 2 & 2 & 4 & 4 & 2 \\
\hline Degree: & 24 & 28 & 30 & 32 & 35 & 36 & 40 & 42 & 48 & 105 \\
Number: & 2 & 2 & 2 & 2 & 2 & 1 & 2 & 1 & 1 & 8 \\
\hline Degree: & 210 & 315 & 420 & 630 & 840 & 1120 & 1344 & 1260 & 1680 & 2240 \\
Number: & 8 & 8 & 6 & 8 & 4 & 8 & 6 & 5 & 1 & 8 \\
\hline Degree: & 2520 & 2688 & 3360 & 4032 & 4480 & 5040 & 5376 & 6720 & 5040 & 8064 \\
Number: & 6 & 2 & 8 & 2 & 2 & 1 & 1 & 4 & 2 & 2 \\
\hline
\end{tabular}


The Alperin weight conjecture and Dade's conjecture for the simple group $\mathrm{J}_{4}$

Table A.17: The degrees of characters in $\operatorname{Irr}\left(6 . M_{22} .2\right)$

\begin{tabular}{r|rrrrrrrrrrrrr}
\hline Degree: & 1 & 10 & 21 & 42 & 45 & 55 & 56 & 90 & 99 & 120 & 132 & 154 & 198 \\
Number: & 2 & 4 & 2 & 1 & 4 & 2 & 2 & 2 & 2 & 2 & 2 & 2 & 1 \\
\hline Degree: & 210 & 231 & 240 & 252 & 308 & 330 & 385 & 420 & 440 & 462 & 560 & 660 & 768 \\
Number: & 6 & 2 & 1 & 3 & 1 & 2 & 2 & 4 & 2 & 2 & 1 & 2 & 2 \\
\hline
\end{tabular}

Table A.18: The degrees of characters in $\operatorname{Irr}\left(\left(3^{2} \times 2^{3}\right) .\left(2 \times S_{3}\right)\right)$

\begin{tabular}{r|rrrrrr}
\hline Degree: & 1 & 2 & 3 & 4 & 6 & 12 \\
Number: & 8 & 8 & 8 & 2 & 12 & 2 \\
\hline
\end{tabular}

Table A.19: The degrees of characters in $\operatorname{Irr}\left(\left(3^{2}: 2 \times 2^{3}\right) . S_{4}\right)$

\begin{tabular}{r|rrrrrrrrr}
\hline Degree: & 1 & 2 & 3 & 4 & 6 & 8 & 12 & 16 & 24 \\
Number: & 4 & 6 & 4 & 2 & 4 & 4 & 1 & 2 & 4 \\
\hline
\end{tabular}

\section{References}

1. J. L. AlPERIN, 'Weights for finite groups', The Arcata Conference on Representations of Finite Groups, Proc. Sympos. Pure Math. 47 (Amer. Math. Soc., Providence, RI, 1987) 369-379. 119

2. JiAnbei An and C. W. EAton, 'The $p$-local rank of a block', J. Group Theory 3 (2000) 369-380. 121

3. Jianbei An and E. A. O'Brien, A local strategy to decide the Alperin and Dade conjectures', J. Algebra 206 (1998) 183-207. 119, 122, 123, 127

4. Jianbei An and E. A. O'Brien, 'The Alperin and Dade conjectures for the simple Fischer group $\mathrm{Fi}_{23}$ ', Internat. J. Algebra Comput. 9 (1999) 621-670. 119, 122, 127

5. R. A. Wilson, et al. 'AtLas of finite group representations', http: / / www . mat.bham.ac.uk/atlas. 121

6. H. I. Blau and G. O. Michler, 'Modular representation theory of finite groups with T. I. Sylow p-subgroups', Trans. Amer. Math. Soc. 319 (1990) 417-468. 123

7. Wieb Bosma, John Cannon and Catherine Playoust, 'The Magma algebra system I: The user language', J. Symbolic Comput. 24 (1997) 235-265. 119

8. J. H. Conway, R. T. Curtis, S. P. Norton, R. A. Parker and R. A. Wilson, Atlas of finite groups (Clarendon Press, Oxford, 1985). 121, 123

9. E. C. DADE, 'Counting characters in blocks, I', Invent. Math. 109 (1992) 187-210. $119,120,123,132$ 
The Alperin weight conjecture and Dade's conjecture for the simple group $\mathrm{J}_{4}$

10. E. C. DADE, 'Counting characters in blocks, II.9', Representation theory offinite groups (Columbus, $\mathrm{OH}, 1995$ ), Ohio State Univ. Math. Res. Inst. Publ. 6 (de Gruyter, Berlin, 1997) 45-59. 119, 120

11. C. W. EAton, 'On finite groups of $p$-local rank one and conjectures of Dade and Robinson', J. Algebra 238 (2001) 623-642. 123, 132

12. The GAP GrouP, 'GAP - groups, algorithms, and programming, Version 4.3' (2002), http: / /www.gap-system.org. 119

13. G. Hiss and K. Lux, Brauer trees of sporadic groups (Oxford Science Publications, 1989). 123, 125

14. P. B. Kleidman and R. A. Wilson, 'The maximal subgroups of $\mathrm{J}_{4}$ ', Proc. London Math. Soc. 56 (1988) 484-510. 119, 122, 123

15. H. NagaO and Y. Tsushima, Representations of finite groups, (Academic Press, Inc., Boston, MA, 1989). 125

16. Robert A. Wilson, 'Standard generators for sporadic simple groups', J. Algebra 184 (1996) 505-515.

17. SAtoshi Yoshiara, 'The radical 2-subgroups of the sporadic simple groups $\mathrm{J}_{4}, \mathrm{Co}_{2}$ and Th', J. Algebra 233 (2000) 309-341. 119, 122, 123, 126, 127

Jianbei An an@math.auckland.ac.nz

E. A. O'Brien obrien@math.auckland.ac.nz

Department of Mathematics

University of Auckland

Auckland

New Zealand

R. A. Wilson R.A.Wilson@bham.ac.uk

Department of Mathematics

The University of Birmingham

Birmingham B15 2TT 